\title{
Effect of cooling condensing cover on the performance of $N$-identical photovoltaic thermal-compound parabolic concentrator active solar still: a comparative study
}

\author{
Poonam Joshi ${ }^{1}$ (D) G. N. Tiwari
}

Received: 30 November 2017 / Accepted: 30 May 2018 / Published online: 21 June 2018

(c) The Author(s) 2018

\begin{abstract}
In this work, photovoltaic thermal-compound parabolic concentrators (PVT-CPC) are integrated to a single slope solar still (SS-SS) through a heat exchanger placed in the basin. A continuous water flow is provided over the condensing cover of SS-SS for yield enhancement. An effect of cooling condensing cover on energy and exergy analysis (thermal and electrical) together with the production cost of distilled water $(₹ / \mathrm{kg})$ has been studied for the following three cases: (I) the proposed partially covered photovoltaic thermal-compound parabolic concentrator single slope solar still (PVT-CPC-SS-SS), (II) fully covered thermal-compound parabolic concentrator single slope solar still (PVT-CPC-SS-SS), and (III) flat plate thermalcompound parabolic concentrator single slope solar still (FPC-CPC-SS-SS). Design parameters have been optimized for maximum distillate output (energy) and exergy on annual performance basis. Moreover, higher daily yield (37.9 kg) is obtained for case (iii). In addition, higher electrical module efficiency (13\%) is obtained for case (ii) for the month of January when the solar cell temperature is $55^{\circ} \mathrm{C}$ at the optimized conditions. However, the proposed system gives daily yield $(35.78 \mathrm{~kg})$ and generates electricity at module efficiency of $12 \%$. The energy payback time of the proposed system is estimated to be 2 years.
\end{abstract}

Keywords Photovoltaic thermal-compound parabolic concentrator (PVT-CPC) · Solar distillation · Heat exchanger . Energy and exergy

$\begin{array}{ll}\text { List of symbols } \\ A & \text { Area }\left(\mathrm{m}^{2}\right) \\ A_{\mathrm{m}} & \text { Module area }\left(\mathrm{m}^{2}\right) \\ A_{\mathrm{g}} & \text { Area of glass }\left(\mathrm{m}^{2}\right) \\ A_{\mathrm{b}} & \text { Area of basin }\left(\mathrm{m}^{2}\right) \\ C_{\mathrm{f}} & \text { Specific heat capacity of fluid (water })\left(\frac{\mathrm{J}}{\mathrm{kg} \mathrm{K}}\right) \\ C_{\mathrm{w}} & \text { Specific heat capacity of water }\left(\frac{\mathrm{J}}{\mathrm{kg} \mathrm{K}}\right) \\ \mathrm{d} x & \text { Elemental section } \\ g & \text { Acceleration due to gravity }\end{array}$

Electronic supplementary material The online version of this article (https://doi.org/10.1007/s40095-018-0276-6) contains supplementary material, which is available to authorized users.

Poonam Joshi

poonam_joshi@aol.com; pjoshi3112@gmail.com

Centre for Energy Studies, Indian Institute of Technology Delhi, Delhi, India

2 Bag Energy Research Society (BERS), SODHA BERS COMPLEX, Plot No. 51, Mahamana Nagar, Karaudi, Varanasi, UP 2210 05, India

$\begin{array}{ll}G r & \text { Grashof Number } \\ E_{\mathrm{x}} & \text { Exergy } \\ h_{\mathrm{rw}} & \text { Radiative heat transfer coefficient }\left(\mathrm{W} / \mathrm{m}^{2} \mathrm{~K}\right) \\ h_{\mathrm{cw}} & \text { Convective heat transfer coefficient }\left(\mathrm{W} / \mathrm{m}^{2} \mathrm{~K}\right) \\ h_{\mathrm{ew}} & \text { Evaporative heat transfer coefficient }\left(\mathrm{W} / \mathrm{m}^{2} \mathrm{~K}\right) \\ h_{\mathrm{bw}} & \text { Heat transfer coefficient from basin to water } \\ & \left(\mathrm{W} / \mathrm{m}^{2} \mathrm{~K}\right) \\ h_{\mathrm{ba}} & \text { Heat transfer coefficient from basin to ambient } \\ & \left(\mathrm{W} / \mathrm{m}^{2} \mathrm{~K}\right) \\ I(t) & \text { Solar radiation }\left(\mathrm{W} / \mathrm{m}^{2}\right) \\ K_{\mathrm{g}} & \text { Thermal conductivity }\left(\frac{\mathrm{w}}{\mathrm{m}} \mathrm{K}\right) \\ L_{\mathrm{g}} & \text { Length of glass }(\mathrm{m}) \\ \dot{m}_{\mathrm{f}} & \text { Mass flow rate of working fluid }(\mathrm{kg} / \mathrm{s}) \\ N & \text { Number of thermal collectors } \\ N u & \text { Nusselt number } \\ P r & \text { Prandlt number } \\ P_{\mathrm{w}} & \text { Partial saturated water vapor at water temperature } \\ & \left(\frac{\mathrm{N}}{\mathrm{m}^{2}}\right) \\ P & \text { Partial saturated water vapor at glass temperature } \\ P_{\mathrm{gi}} & \left(\frac{\mathrm{N}}{\mathrm{m}^{2}}\right) \\ & \end{array}$


$\dot{Q}_{\mathrm{u}, N} \quad$ Rate of useful energy (W)

$r_{1} \quad$ Inner radius of heat exchanger (m)

$r_{2} \quad$ Outer radius of heat exchanger (m)

$t \quad$ Time

$T \quad$ Temperature $\left({ }^{\circ} \mathrm{C}\right)$

$T_{\mathrm{a}} \quad$ Ambient temperature $\left({ }^{\circ} \mathrm{C}\right)$

$T_{\text {foN }} \quad$ Outlet fluid temperature $\left({ }^{\circ} \mathrm{C}\right)$

$T_{\mathrm{w}} \quad$ Water temperature $\left({ }^{\circ} \mathrm{C}\right)$

$T_{\mathrm{wf}} \quad$ Temperature of water flowing above condensing cover $\left({ }^{\circ} \mathrm{C}\right)$

$T_{\mathrm{fi}} \quad$ Inlet water temperature $\left({ }^{\circ} \mathrm{C}\right)$

$T_{\mathrm{gi}} \quad$ Inner glass temperature $\left({ }^{\circ} \mathrm{C}\right)$

$T_{\mathrm{go}} \quad$ Outer glass temperature $\left({ }^{\circ} \mathrm{C}\right)$

$U$ Overall heat transfer coefficient $\left(\mathrm{W} / \mathrm{m}^{2} \mathrm{~K}\right)$

\section{Subscript}

$\begin{array}{ll}\text { a } & \text { Ambient air } \\ \text { eff } & \text { Effective } \\ \mathrm{g} & \text { Glass } \\ \mathrm{w} & \text { Water }\end{array}$

\section{Greek letters}

$\alpha \quad$ Absorptivity

$\alpha_{\mathrm{b}} \quad$ Absorptivity of basin

$\alpha_{\mathrm{w}} \quad$ Absorptivity of water

$(\alpha \tau)_{\text {eff }}$ Product of effective absorptivity and

$\tau \quad$ Transmissivity

$\eta_{\mathrm{m}} \quad$ Module efficiency

\section{Introduction}

Water is crucial to sustain life on earth, and its demand is primarily influenced by climate change, population growth and urbanization, and energy security policies. Last few decades have seen a sharp decline in the availability of potable water and it is projected that the world will face a 20\% water deficit by 2030 [1]. World Health Organization (WHO), World Bank (WB), World Wildlife Fund (WWF), World Water Council (WWC), United Nation Development Program (UNDP), etc., have worked extensively to promote water conservation by regulation and implementation of water policies. This clearly indicates that access to potable water continues to be a major problem.

To address the global crisis of water availability, solar desalination/distillation promises to be an effective technology. Solar still (passive/active) harness solar energy (emission free), which distillates brackish water $(\approx 10,000) \mathrm{ppm}$ and saline water $(\approx 45,000) \mathrm{ppm}$ into potable water. A first solar distillation apparatus was described by Giovanni Battista Della Porta (1535-1615) [2]. After him many researchers worked on different designs (Table 1) of a solar still to improve the performance of the system on an hourly and annual basis to obtain the increased quantity of potable water. However, the performance of a solar still is limited by the temperature difference $(\Delta T)$ between condensing and evaporating areas. A continuous flow of water or air over condensing cover leads to cooling, which increases the temperature difference and improves productivity (Table 1). In addition, it cleans the dirt and filth on the condensing cover, which otherwise, reduces the solar still (SS) efficiency. Table 1 also includes previous work done for the increase in yield, efficiency, and productivity of a solar still because of cooling the condensing cover.

Previous studies (Table 1) on passive and active solar stills show that active solar still have higher productivity as they are integrated to external heat sources, e.g., flat plate collector, evacuated tubular collector, solar concentrators, photovoltaic thermal flat plate collector, and photovoltaic thermal-compound parabolic concentrator, which preheats the saline or brackish water. Flat plate collectors integrated with solar stills are commercialized, but are not self-sustainable. For a self-sustainable active solar distillation system, electrical energy is required to operate a pump/motor, which overcomes the pressure drop in connecting insulated pipes. So, the integration of the semitransparent photovoltaic module is a novel idea.

Integration of photovoltaic thermal-compound parabolic concentrators (PVT-CPC) with a solar still concentrates global solar radiation into photovoltaic thermal collector, which heats the working fluid and additionally generates electricity (direct current). Recently, Singh and Tiwari [28] did a comparative performance analysis of solar stills (single and double slope solar still) integrated with PVT-CPC and performed an exergo, enviro-economic, and productivity analysis. They have concluded that active single slope solar still (SS-SS) gives 3\% higher daily overall thermal efficiency and $2 \%$ higher daily productivity if the depth of water in the basin is $0.56 \mathrm{~m}$. In their work, the working fluid in PVT-CPC collectors is brackish/saline water, which reduces the life of PVT-CPC collectors due to corrosion. To overcome this problem, in our proposed system, a helically coiled heat exchanger (copper) is placed inside the SS-SS. The heat exchanger is integrated to the thermal collectors [Type (a-c)] to make a closed loop, as shown in Fig. 1a-c.

Type (a) Partially covered photovoltaic thermal-compound parabolic concentrator (PVT-CPC) $\left(A_{\mathrm{rm}}=0.25 \mathrm{~m}^{2}\right.$, $A_{\mathrm{rc}}=0.65 \mathrm{~m}^{2}$ ), proposed system

Type (b) Fully covered photovoltaic thermal-compound parabolic concentrator (PVT-CPC) $\left(A_{\mathrm{rm}}=1 \mathrm{~m}^{2}, A_{\mathrm{rc}}=0 \mathrm{~m}^{2}\right)$

Type (c) Flat plate collector-compound parabolic concentrator (FPC-CPC) $\left(A_{\mathrm{rm}}=0 \mathrm{~m}^{2}, A_{\mathrm{rc}}=1 \mathrm{~m}^{2}\right)$

In the proposed cases (I-III) the water mass (brackish water) and the working fluid (water) is used to obtain higher yield, which have not been considered earlier. The three cases (I-III), which are considered for the study are: 


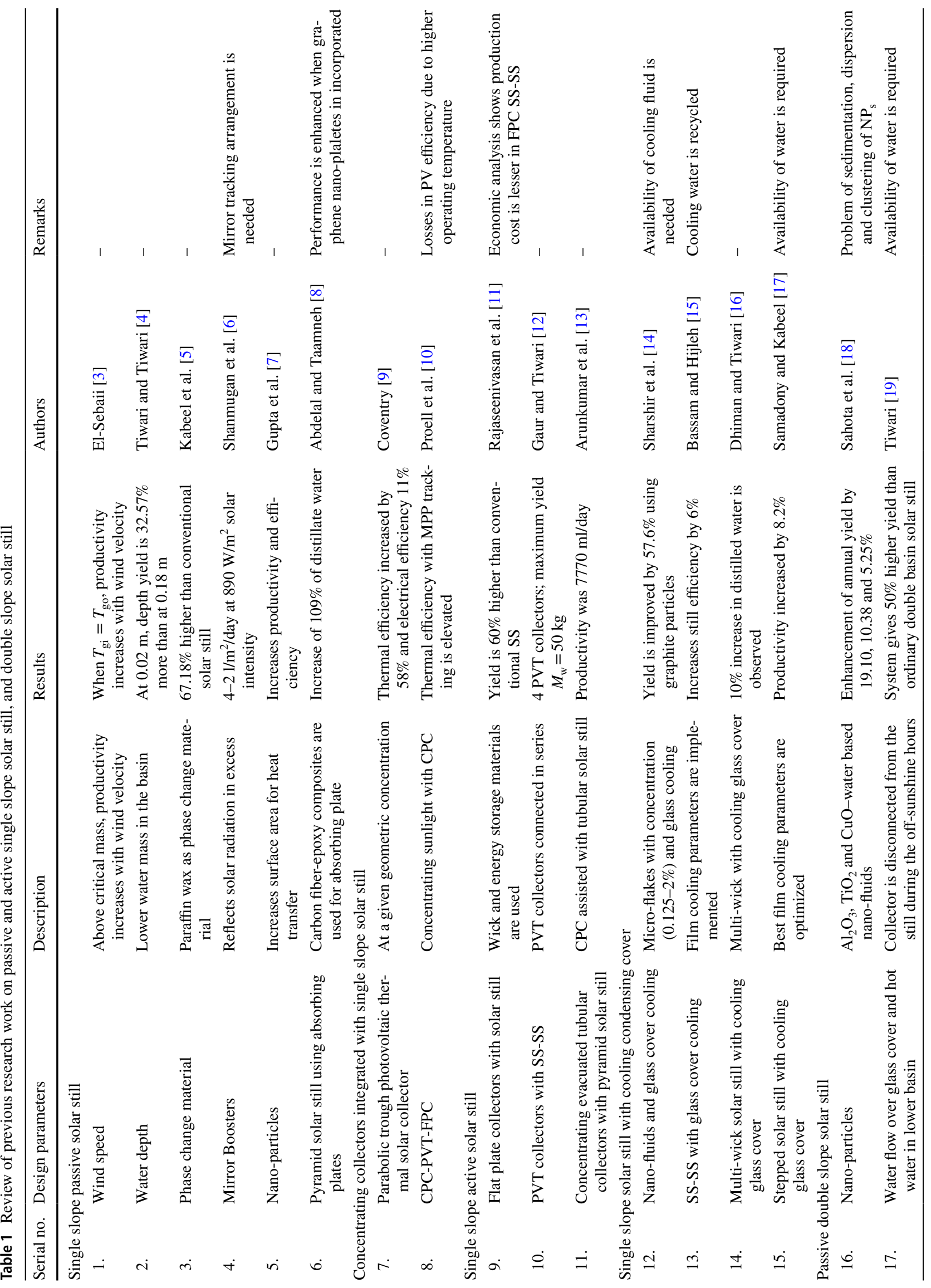




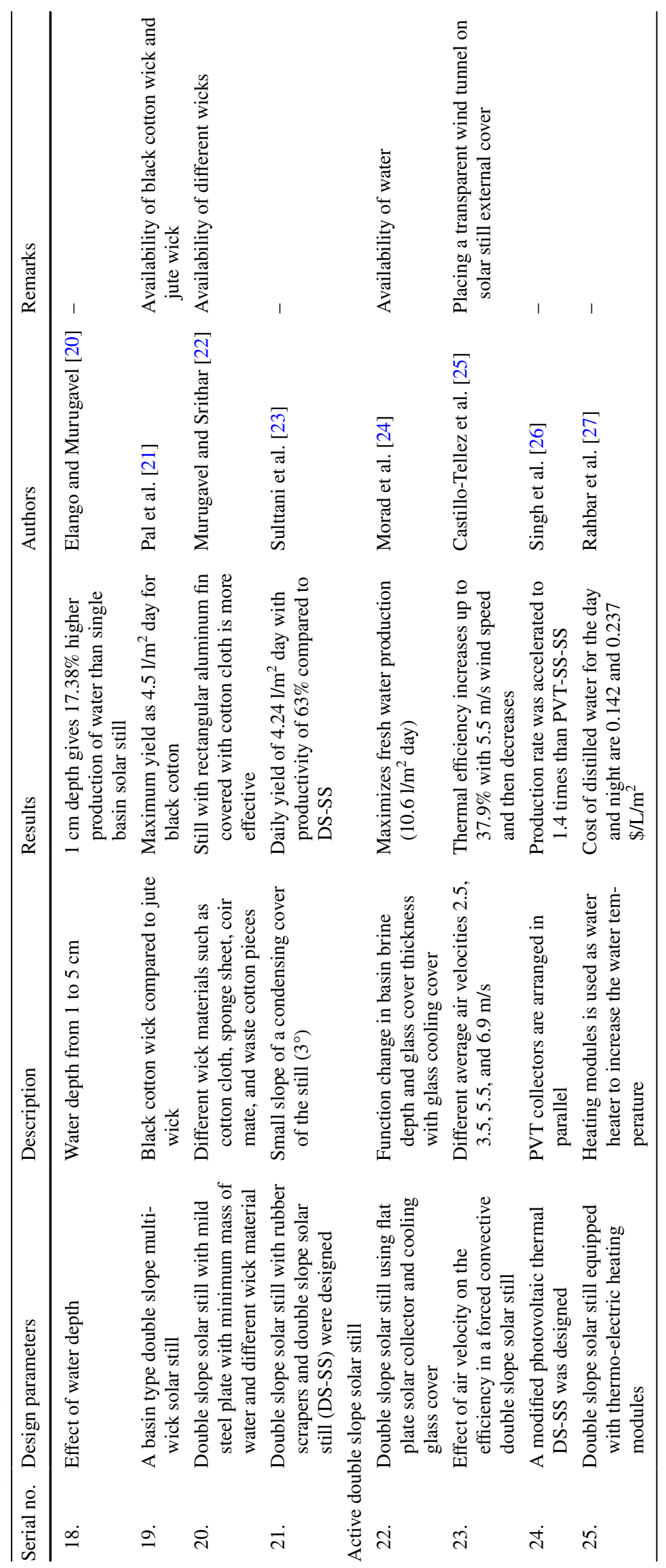


Fig. 1 a Helically coiled copper heat exchanger. b Schematic view of an active single slope solar still integrated with the help of a heat exchanger [case (I)]. c Schematic view of an active single slope solar still integrated with the help of a heat exchanger [case (II)]. d Schematic view of an active single slope solar still integrated with the help of a heat exchanger [case (III)]. e Schematic view of partially covered photovoltaic thermal flat plate collector (PVT-FPC) integrated with a single slope solar still (a)

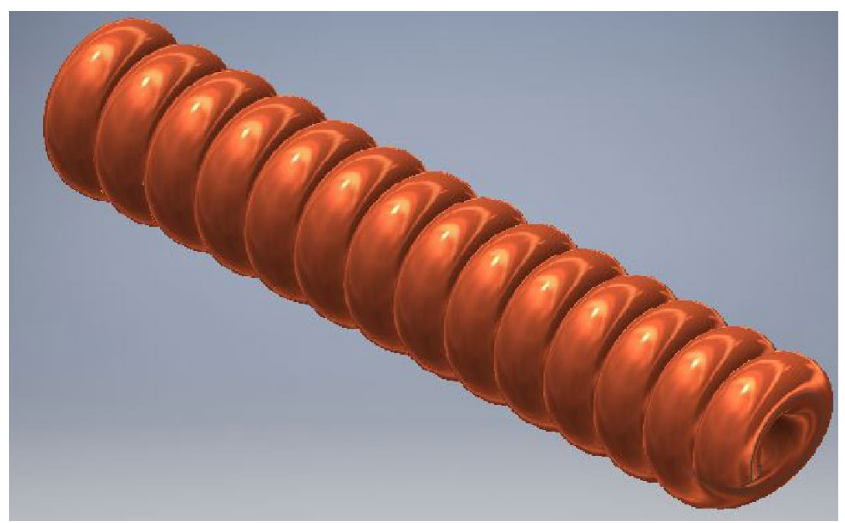

(b)

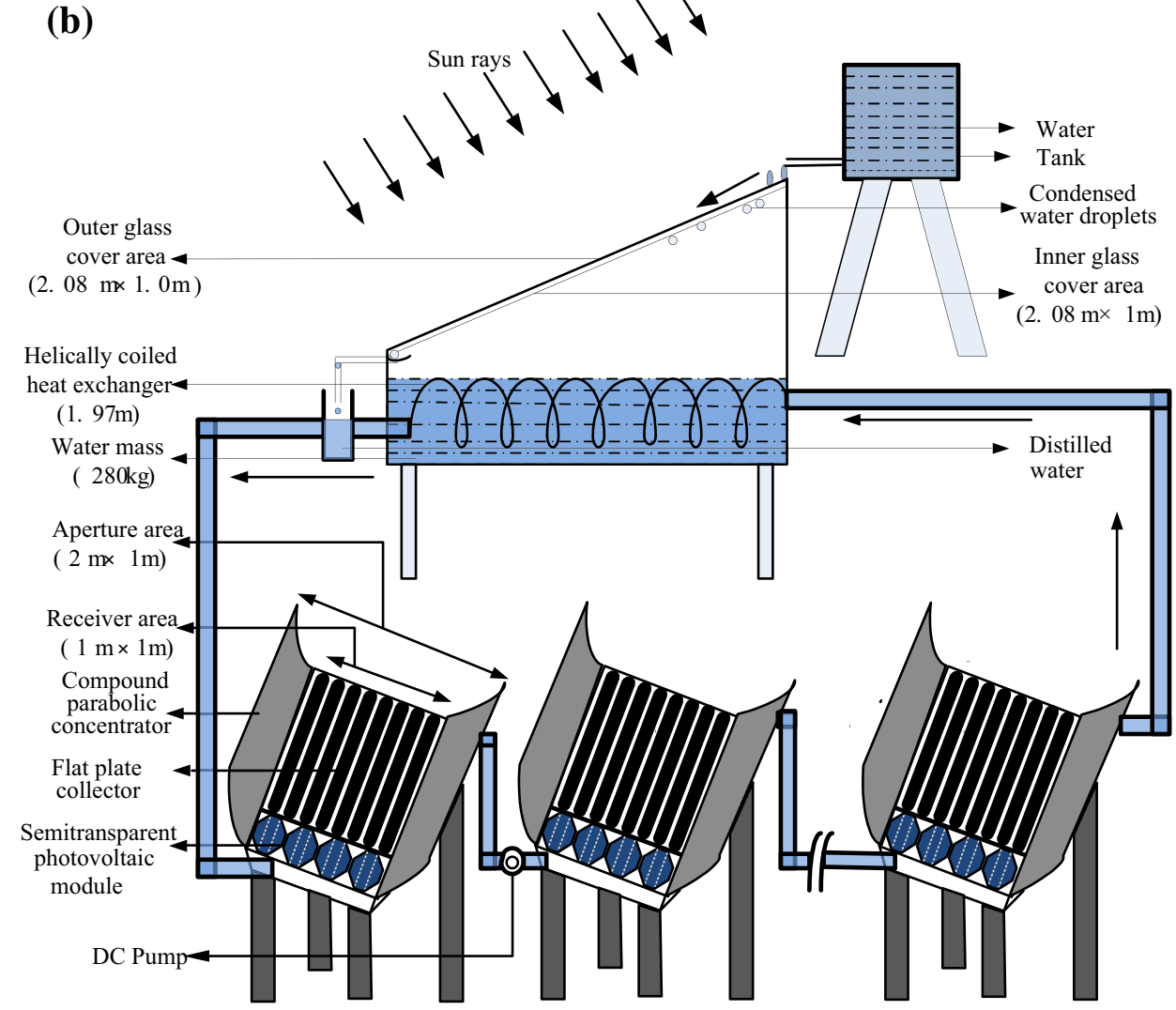

Case (I) Partially covered photovoltaic thermal-compound parabolic concentrator single slope solar still (PVT-CPC-SS-SS),

Case (II) Fully covered thermal-compound parabolic concentrator single slope solar still (PVT-CPC-SS-SS)

Case (III) Flat plate thermal-compound parabolic concentrator single slope solar still (FPC-CPC-SS-SS).

In addition, if cooling condensing cover over SS-SS with heat exchanger and thermal collector Type $(\mathrm{a}-\mathrm{c})$ are taken into account it will lead to higher distilled water as well as the life of solar thermal collectors will increase, which will make the system more viable economically.
As Singh and Tiwari [28] have not studied the effect of heat exchanger and cooling condensing cover of SS-SS. This paper deals with the energy and exergy analysis of the three active solar distillation units [cases (I-III)] for the optimum design. Moreover, energy matrices, production cost of distilled water and electricity, and co-generation efficiency have been analyzed and compared among three cases (I-III). 
Fig. 1 (continued)

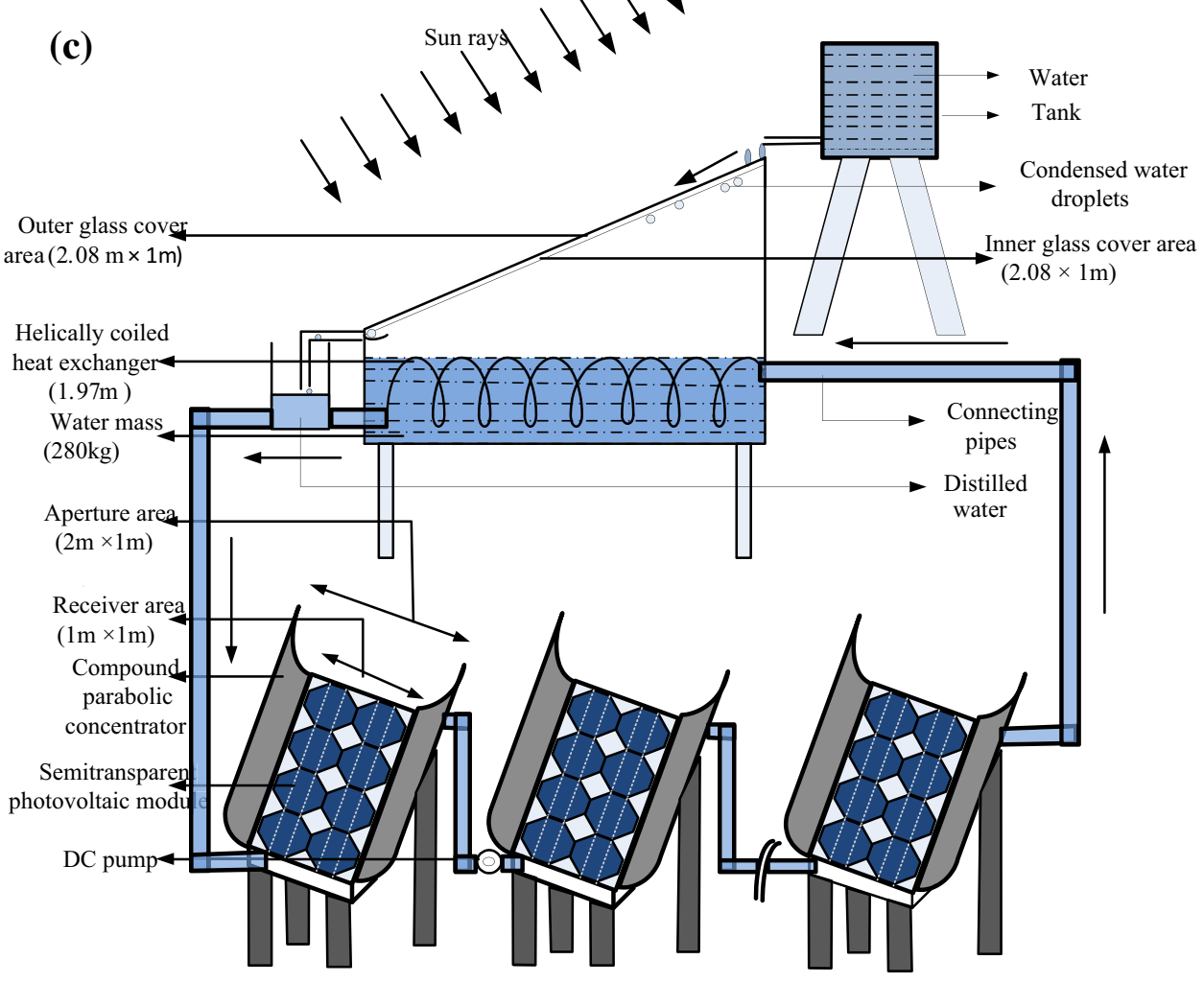

(d)

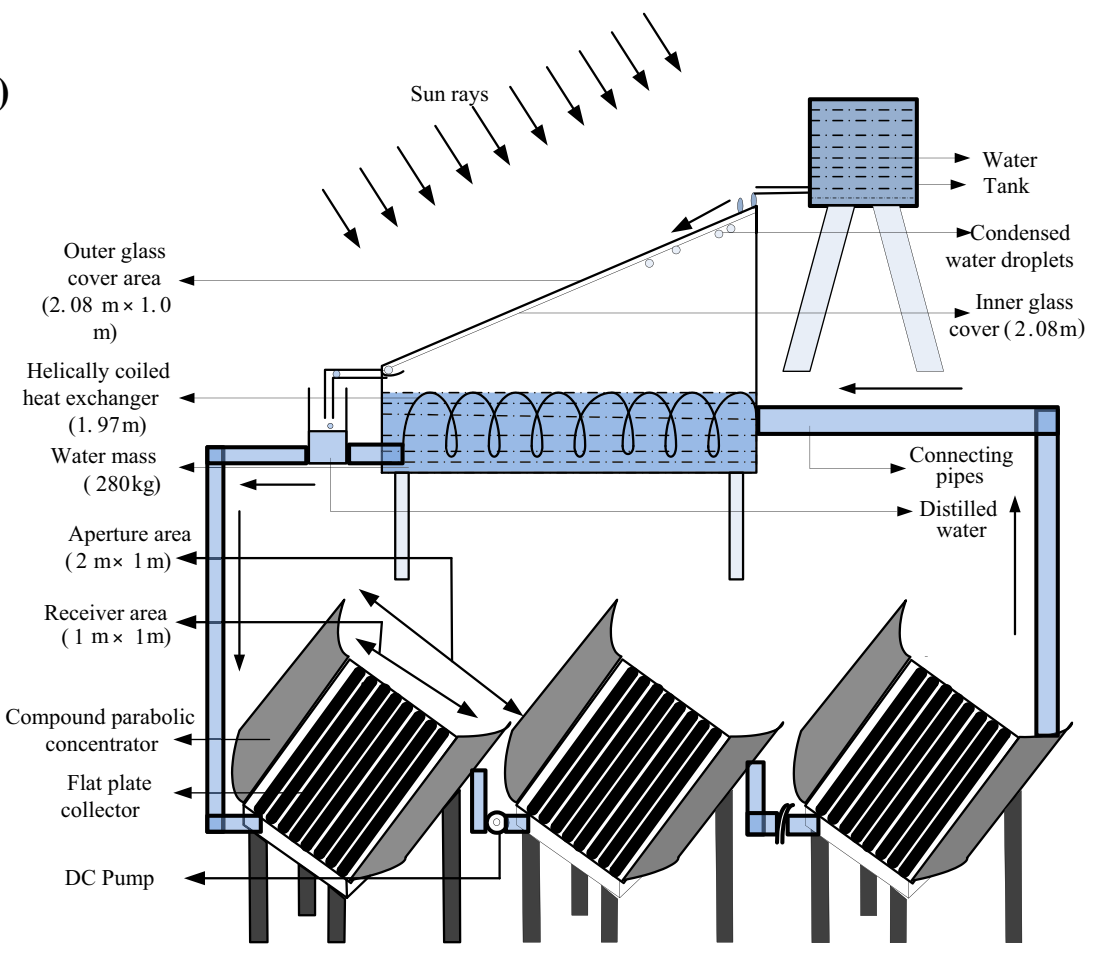


Fig. 1 (continued)

\section{System description}

The concentration ratio $(c)$ of compound parabolic concentrator made up of (aluminum) for a given acceptance angle $\left(\theta_{\mathrm{c}}=30^{\circ}\right)$ can be calculated from the following equation:

$c=\frac{1}{\sin 2 \theta_{\mathrm{c}}}$.

Rabl [29] derived an expression for aperture width, height, and arc length for the truncated compound parabolic concentrator (CPC). The side view of helically coiled copper heat exchanger (optimized numerically) with a length of $1865 \mathrm{~mm}$ having 14-helix with a pitch of $140 \mathrm{~mm}$ is shown in Fig. 1a. Figure 1b-d depicts three active solar still systems, i.e., PVT-CPC integrated with a SS-SS with the help of a heat exchanger. The CPC is inclined at $30^{\circ}$ (latitude of New Delhi, India) toward south to receive the maximum annual global solar radiation $\left(I_{\mathrm{t}}\right)$, and concentrates global radiation falling on an aperture area towards the receiver area $\left(I_{\mathrm{b}}\right)$ for the three different configurations [Type $\left.(\mathrm{a}-\mathrm{c})\right]$. Thus, the fluid flowing beneath the photovoltaic thermalcompound parabolic collectors (PVT-CPC) Type (a-c) gets heated up at a faster rate compared to the photovoltaic thermal flat plate collector (PVT-FPC). Type (a) consists of a semitransparent PV module in the lower portion, and a flat plate collector at the upper portion of the receiver area and outlet from the semitransparent PV module is the inlet to the tube-in-flat plate collectors (Fig. 1b). This arrangement gives increased electrical efficiency because initially higher heat (generated by solar cells) is extracted by the fluid (water) flowing at the rear portion of PVT-CPC. Above-mentioned configurations [Type $(\mathrm{a}-\mathrm{c})]$ are arranged in series (to increase the water temperature), i.e., the outlet of first PVT-CPC is an inlet to the second and continues till $N$ th PVT - CPC to obtain maximum outlet fluid temperature and yield (Eqs. 8 and 21). The pressure drop in insulated

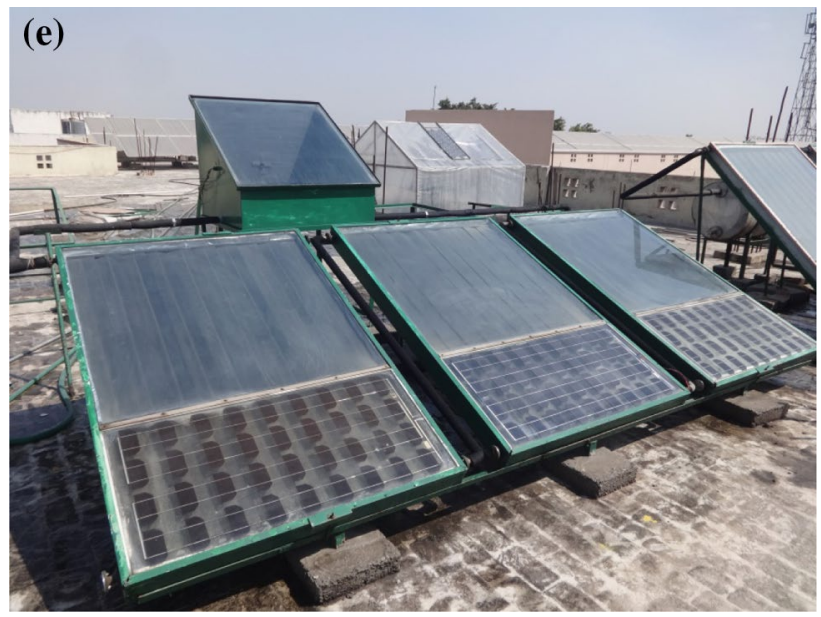

connecting pipes is overcome by DC motor driven by the semitransparent PV modules.

The outlet of Nth PVT-CPC is connected to the basin of SS-SS with the help of helically coiled copper heat exchanger, and the outlet from SS-SS is connected to the first PVT-CPC. Thus, water circulates in a closed loop. The absorber plate (black dye-in-water solution) is placed in the SS-SS made up of fiber-reinforced plastic (FRP) having an effective basin area of $2 \mathrm{~m}^{2}$. Cooling condensing cover has a thickness of $0.004 \mathrm{~m}$ inclined at an angle of $15^{\circ}$ with the horizontal. Glass is recommended as a cooling condensing cover over plastic. The side walls are blackened so that maximum solar flux is absorbed inside the SS-SS. The whole system is sealed with the window putty and fixed on an iron stand. Table 2 gives detail specifications for three active SS-SS, cases (I-III), and thermal collectors Type (a-c).

\section{Experimental validation}

We have carried out experimental validation for a special case, i.e., SS-SS integrated with photovoltaic thermal flat plate collectors (PVT-FPCs) without heat exchanger. Experimental setup is shown in Fig. 1e. SS-SS made up of fiberreinforced plastic has an effective area of $1 \mathrm{~m}^{2}$, which is connected to three PVT-FPC through an insulated pipe. A DC motor has been used in a closed loop for forced mode of operation. The design parameters of the system are given in Table 3. Concentrating solar radiation $(I(t))$ into PVT-FPC, cooling of condensing cover and integration of helical coiled heat exchanger are excluded from the experimental unit. As the CPC is not included in the design the solar radiation falling on PVT-FPC is global radiation, which has lower intensity than beam radiation. In addition, cooling condensing cover is also removed, which reduces the temperature difference between glass and water in the basin, as a result the yield reduces. However, as the helical heat exchanger is 
Table 2 Specifications of the hybrid active solar distillation systems, case (I-III)

\begin{tabular}{|c|c|}
\hline Components & Specifications \\
\hline \multicolumn{2}{|l|}{ Specifications for single slope solar still } \\
\hline Length & $2 \mathrm{~m}$ \\
\hline Breadth & $1 \mathrm{~m}$ \\
\hline Inclination of glass cover & $15^{\circ}$ \\
\hline Material of body & Fiber-reinforced plastic (FRP) \\
\hline Material of stand & Galvanized iron \\
\hline Condensing material & Glass \\
\hline Orientation & South \\
\hline \multicolumn{2}{|l|}{ Specifications of thermal collectors } \\
\hline Type and number of collectors & Tube in plate type \\
\hline Thickness of copper tubes & $1 \mathrm{~m}$ \\
\hline Tube diameter & $0.0125 \mathrm{~m}$ \\
\hline Angle of CPC with horizontal & $30^{\circ}$ \\
\hline Thickness of CPC & $0.004 \mathrm{~m}$ \\
\hline Aperture area & $2 \mathrm{~m}^{2}$ \\
\hline Receiver area & $1 \mathrm{~m}^{2}$ \\
\hline Aperture area of module [Type (a), Type (b) and Type (c)] & $0.5,2$ and $0 \mathrm{~m}^{2}$ \\
\hline Aperture area of receiver [Type (a),Type (b) and Type (c)] & $1.5,0$ and $2 \mathrm{~m}^{2}$ \\
\hline Receiver area of module [Type (a), Type (b) and Type (c)] & $0.25,1$ and $0 \mathrm{~m}^{2}$ \\
\hline Receiver area of collector [Type (a), Type (b) and Type (c)] & $0.75,0$ and $1 \mathrm{~m}^{2}$ \\
\hline Specific heat capacity of fluid & $4190 \mathrm{~J} / \mathrm{kg} \mathrm{K}$ \\
\hline$K_{\mathrm{g}}$ & $0.78 \mathrm{~W} / \mathrm{m}^{2}{ }^{\circ} \mathrm{C}$ \\
\hline$L_{\mathrm{g}}$ & $0.003 \mathrm{~m}$ \\
\hline$K_{\mathrm{i}}$ & $0.166 \mathrm{~W} / \mathrm{m}^{2}{ }^{\circ} \mathrm{C}$ \\
\hline$L_{\mathrm{i}}$ & $0.1 \mathrm{~m}$ \\
\hline$U_{\mathrm{tc}, \mathrm{p}}$ & $2.67 \mathrm{~W} / \mathrm{m}^{2}{ }^{\circ} \mathrm{C}$ \\
\hline$U_{\mathrm{tc}, \mathrm{a}}$ & $19.34 \mathrm{~W} / \mathrm{m}^{2}{ }^{\circ} \mathrm{C}$ \\
\hline$P F_{1}$ & 0.12 \\
\hline$P F_{2}$ & 0.58 \\
\hline$P F_{\mathrm{c}}$ & 0.98 \\
\hline$h_{\mathrm{pf}}$ & $100 \mathrm{~W} / \mathrm{m}^{2}{ }^{\circ} \mathrm{C}$ \\
\hline$h_{\mathrm{i}}$ & $2.8 \mathrm{~W} / \mathrm{m}^{2}{ }^{\circ} \mathrm{C}$ \\
\hline$\rho$ & 0.84 \\
\hline$\tau_{\mathrm{g}}$ & 0.95 \\
\hline$\alpha_{\mathrm{c}}$ & 0.7 \\
\hline$\beta_{\mathrm{c}}$ & 0.8 \\
\hline$\alpha_{\mathrm{p}}$ & 0.6 \\
\hline$F^{\prime}$ & 0.96 \\
\hline$\eta_{0}$ & 0.15 \\
\hline
\end{tabular}

removed there is an increase in temperature of water $\left(T_{\mathrm{w}}\right)$ as there is direct transfer of hot water in the basin of solar still. The daily yield ( $\left.\dot{m}_{\text {ew }}\right)$ obtained from PVT-FPC SS-SS is $3.85 \mathrm{~kg}$ for three PVT-FPC, which is lesser that the yield obtained from the proposed system [case (I)], i.e., $32.46 \mathrm{~kg}$ for the month of February.

\section{Thermal model}

The assumptions considered for the thermal modeling for different components of the active solar distillation systems to reduce complexity of the system are as follows: 
Table 3 Design parameters of the photovoltaic thermal flat plate collector single slope solar still

Specifications of PVT-FPC active single slope solar still

(a) Specifications of single slope solar still

$\begin{array}{ll}\text { Length } & 1 \mathrm{~m} \\ \text { Breadth } & 1 \mathrm{~m} \\ \text { Inclination of glass cover } & 30^{\circ} \\ \text { Thickness of glass cover } & 0.004 \mathrm{~m} \\ \text { (b) Specifications of photovoltaic module } & \\ \text { Area of single solar cell } & 0.007 \mathrm{~m}^{2} \\ \text { Area of PV module } & 0.25 \mathrm{~m}^{2} \\ \text { Efficiency of PV module } & 12 \% \\ \text { Maximum power rating } & 40 \mathrm{~W} \\ \propto_{\mathrm{c}} & 0.9 \\ \eta_{\mathrm{o}} & 0.15 \\ \beta_{\mathrm{o}} & 0.0045 /{ }^{\circ} \mathrm{C} \\ \beta_{\mathrm{c}} & 0.89\end{array}$

(c) Specifications of flat plate collector

\begin{tabular}{ll}
$\tau_{\mathrm{g}}$ & 0.9 \\
$\propto_{\mathrm{p}}$ & 0.8 \\
$L_{\mathrm{g}}$ & $0.003 \mathrm{~m}$ \\
$K_{\mathrm{g}}$ & $0.816 \mathrm{~W} / \mathrm{mK}$ \\
$L_{\mathrm{i}}$ & $0.1 \mathrm{~m}$ \\
$K_{\mathrm{i}}$ & $0.166 \mathrm{~W} / \mathrm{mK}$ \\
$L_{\mathrm{p}}$ & $0.002 \mathrm{~m}$ \\
$K_{\mathrm{p}}$ & $64 \mathrm{~W} / \mathrm{mK}$ \\
$C_{\mathrm{f}}$ & $4179 \frac{\mathrm{J}}{\mathrm{kg} \mathrm{K}}$ \\
$\rho$ & $1000 \mathrm{~kg} / \mathrm{m}^{3}$ \\
Area of collector $A_{\mathrm{c}}$ & $1.75 \mathrm{~m}{ }^{2}$ \\
$h_{\mathrm{i}}$ & $5.8 \mathrm{~W} / \mathrm{m}^{2} \mathrm{~K}$ \\
$h_{\mathrm{o}}$ & $9.5 \mathrm{~W} / \mathrm{m}^{2} \mathrm{~K}$ \\
Tube diameter & $0.0125 \mathrm{~m}$ \\
\hline
\end{tabular}

1. The SS-SS and thermal collectors are in quasi-steady state.

2. Constant water mixing is preferred; thus, no stratification of water occurs in the basin of a SS-SS.

3. Ohmic losses in solar cells are neglected.

4. Heat capacity of the glass, solar cell, absorbing and insulating material used in the thermal collectors and SS-SS, and water flowing over the glass cover has been neglected.

5. The temperature gradients along the condensing cover thickness and water film have not been taken into account.

6. In SS-SS, film-type condensation occurs throughout the glass.

Energy balance equations for cooling condenser cover active SS-SS with helical heat exchanger [case (I)] are written as follow.
For water flowing over condensing cover:

$$
\dot{m}_{\mathrm{f} 1} C_{\mathrm{f}} \frac{\mathrm{d} T_{\mathrm{f}}}{\mathrm{d} x} \mathrm{~d} x=\left[h_{1}\left(T_{\mathrm{go}}-T_{\mathrm{wf}}\right)-h_{\mathrm{o}}\left(T_{\mathrm{wf}}-T_{\mathrm{a}}\right)\right] b \mathrm{~d} x .
$$

For outer glass cover and inner glass cover:

$$
\begin{aligned}
& h_{\mathrm{kg}}\left(T_{\mathrm{gi}}-T_{\mathrm{go}}\right) A_{\mathrm{g}}=h_{1}\left(T_{\mathrm{go}}-T_{\mathrm{wf}}\right) A_{\mathrm{g}} \\
& \alpha_{\mathrm{g}} I(t) A_{\mathrm{g}}+h_{2}\left(T_{\mathrm{w}}-T_{\mathrm{gi}}\right) A_{b}=h_{\mathrm{kg}}\left(T_{\mathrm{gi}}-T_{\mathrm{go}}\right) A_{\mathrm{g}} .
\end{aligned}
$$

For water mass in the basin:

$$
\dot{Q}_{\mathrm{u}, N}+\alpha_{\mathrm{w}} I(t) A_{\mathrm{b}}+h_{\mathrm{bw}}\left(T_{\mathrm{b}}-T_{\mathrm{w}}\right) A_{\mathrm{b}}=M_{\mathrm{w}} C_{\mathrm{w}} \frac{\mathrm{d} T_{\mathrm{w}}}{\mathrm{d} t}+h_{2}\left(T_{\mathrm{w}}-T_{\mathrm{gi}}\right) A_{\mathrm{b}} .
$$

\section{Useful energy gain from Nth-PVT-CPC arranged in series combination}

The energy balance equation for helical heat exchanger immersed in the basin of the SS-SS is given by

$\dot{m}_{\mathrm{f}} C_{\mathrm{f}} \frac{\mathrm{d} T_{\mathrm{f}}}{\mathrm{d} x} \mathrm{~d} x=-2 \pi r_{1} U\left(T_{\mathrm{f}}-T_{\mathrm{w}}\right)$.

Following boundary conditions is considered

$T_{\mathrm{w}}(x=0)=T_{\mathrm{fo} N} \quad$ and $\quad T_{\mathrm{w}}(x=L)=T_{\mathrm{fi}}$.

Integrating Eq. 6 for the above mentioned boundary conditions, one can get

$T_{\mathrm{fi}}=T_{\mathrm{fo} N} \exp \left(\frac{-2 \pi r_{1} U L}{\dot{m}_{\mathrm{f}} C_{\mathrm{f}}}\right)+T_{\mathrm{w}}\left(1-\exp \left(\frac{-2 \pi r_{1} U L}{\dot{m}_{\mathrm{f}} C_{\mathrm{f}}}\right)\right)$,

where

$U=\left[\frac{1}{h_{\mathrm{w}}}+\left(\frac{r_{1}}{K_{1}}\right) \log \left(\frac{r_{2}}{r_{1}}\right)+\left(\frac{r_{1}}{r_{2}}\right) \frac{1}{h_{\mathrm{w}}}\right]^{-1}$.

Outlet fluid temperature $\left(T_{\mathrm{fo} N}\right)$ for $N$ thermal collector cases $(\mathrm{a}-\mathrm{c})$ can be obtained from the following equation:

$$
\begin{aligned}
T_{\mathrm{fo} N}= & \left(\frac{I_{\mathrm{b}}}{\dot{m}_{\mathrm{f}} C_{\mathrm{f}}}\left(A F_{\mathrm{R}}(\alpha \tau)_{1}\right)\left(\frac{1-K_{\mathrm{K}}^{N}}{1-K_{\mathrm{K}}^{N}}\right)\right) \\
& +\left(\frac{T_{\mathrm{a}}}{\dot{m}_{\mathrm{f}} C_{\mathrm{f}}}\left(A F_{\mathrm{R}} U_{\mathrm{L}}\right)_{1}\left(\frac{1-K_{\mathrm{K}}^{N}}{1-K_{K}^{N}}\right)\right)+\left(T_{\mathrm{fi}} K_{\mathrm{K}}^{N}\right) .
\end{aligned}
$$

The rate of useful thermal energy obtained from thermal collectors can be calculated in Watt (W) from

$\dot{Q}_{\mathrm{u}, N}=\dot{m}_{\mathrm{f}} C_{\mathrm{f}}\left(T_{\mathrm{foN}}-T_{\mathrm{fi}}\right)$.

Substituting $T_{\text {fo } N}$ from Eq. 8 and rearranging Eq. 9 one can get 


$$
\begin{aligned}
T_{\mathrm{fo} N}-T_{\mathrm{fi}}= & \frac{I_{\mathrm{b}}\left(\frac{\left(A F_{\mathrm{R}}(\alpha \tau)\right)_{1}}{\dot{m}_{\mathrm{f}} C_{\mathrm{f}}}\right) \frac{\left(1-K_{\mathrm{K}}^{N}\right)}{1-K_{\mathrm{K}}^{N} F R_{2}} F R_{1}}{\left(1-K_{\mathrm{K}}\right)} \\
& +\frac{T_{\mathrm{a}}\left(\frac{\left(A F_{\mathrm{R}} U_{\mathrm{L}}\right)_{1}}{\dot{m}_{\mathrm{f}} C_{\mathrm{f}}}\right) \frac{\left(1-K_{\mathrm{K}}^{N}\right)}{\left(1-K_{\mathrm{K}}\right)} F R_{1}}{1-K_{\mathrm{K}}^{N} F R_{2}} \\
& -\frac{T_{\mathrm{w}}\left(1-K_{\mathrm{K}}^{N}\right) F R_{1}}{\left(1-K_{\mathrm{K}}^{N} F R_{2}\right)} .
\end{aligned}
$$

Rate of useful thermal energy from Eq. 9 for case (i) can be expressed as

$$
\begin{aligned}
\dot{Q}_{\mathrm{u}, N}= & \frac{I_{\mathrm{b}}\left(A F_{\mathrm{R}}(\alpha \tau)\right)_{1}\left(1-K_{\mathrm{K}}^{N}\right) F R_{1}}{\left(1-K_{\mathrm{K}}\right)\left(1-K_{\mathrm{K}}^{N} F R_{2}\right)} \\
& +\frac{T_{\mathrm{a}}\left(1-K_{\mathrm{K}}^{N}\right)\left(\dot{m}_{\mathrm{f}} C_{\mathrm{f}}\right) F R_{1}}{\left(1-K_{\mathrm{K}}^{N} F R_{2}\right)} \\
& -\frac{T_{\mathrm{w}}\left(1-K_{\mathrm{K}}^{N}\right)\left(\dot{m}_{\mathrm{f}} C_{\mathrm{f}}\right) F R_{1}}{\left(1-K_{\mathrm{K}}^{N} F R_{2}\right)},
\end{aligned}
$$

where $\left(A F_{\mathrm{R}}(\alpha \tau)\right)_{1},\left(A F_{\mathrm{R}} U_{\mathrm{L}}\right)_{1}, F R_{1}, F R_{2}$, and $K_{\mathrm{K}}$ are given in "Appendix".

For basin liner:

$\alpha_{\mathrm{b}} I(t) A_{\mathrm{b}}=h_{\mathrm{bw}}\left(T_{\mathrm{b}}-T_{\mathrm{w}}\right) A_{\mathrm{b}}+h_{\mathrm{ba}}\left(T_{\mathrm{b}}-T_{\mathrm{a}}\right) A_{\mathrm{b}}$.

Solving Eqs. (2-4) one can get

$\frac{\mathrm{d} T_{\mathrm{wf}}}{\mathrm{d} x}+a_{1} T_{\mathrm{wf}}=f(t)_{1}$.

The solution of first-order differential Eq. 13 can be written as

$T_{\mathrm{wf}}=\frac{\overline{f(t)_{1}}}{a_{1}}\left(1-\exp \left(-a_{1} x\right)\right)+T_{\mathrm{wfi}} \exp \left(-a_{1} x\right)$.

Integrating Eq. 14 along the length of glass $x=l$

$\overline{T_{\mathrm{wf}}}=\frac{\overline{f(t)_{1}}}{a_{1}}\left(1-\frac{\left(1-\exp \left(-a_{1} l\right)\right)}{a_{1} l}\right)+T_{\mathrm{wfi}} \frac{1-\exp \left(-a_{1} l\right)}{-a_{1} l}$, where $T_{\text {wfi }}$ is the inlet water temperature flowing over the condensing cover, and $\overline{f(t)_{1}}$ is the average value betweenlength $l=0$ to $l$ where

$f(t)_{1}=\left(\frac{I(t)_{2}+(U A)_{\mathrm{w}, \mathrm{wf}} T_{\mathrm{w}} b+h_{\mathrm{o}} b T_{\mathrm{a}}}{\dot{m}_{\mathrm{f} 1} C_{\mathrm{f}}}\right) ; \quad a_{1}=\frac{\left(U A_{\mathrm{w}, \mathrm{wf}} b\right)+\left(h_{\mathrm{o}} b\right)}{\dot{m}_{\mathrm{f} 1} C_{\mathrm{f}}}$.

Solving Eqs. 3 and 4 with the help of Eq. 15 and substitut$\operatorname{ing} T_{\mathrm{gi}}, T_{\mathrm{b}}$, and $\dot{Q}_{\mathrm{u}, N}$ in Eq. 5 one can get

$T_{\mathrm{w}}=\frac{\overline{f(t)_{2}}}{a_{2}}\left(1-\exp \left(-a_{2} \Delta t\right)\right)+T_{\mathrm{wo}} \exp \left(-a_{2} \Delta t\right)$,

where $f(t)_{2}=\frac{\left(A_{\mathrm{b}}(T)+W(Y(Z+U 1)+V)+X\right)}{M_{\mathrm{w}} C_{\mathrm{w}}}$ and

$$
\begin{aligned}
a_{2}= & \frac{T_{\mathrm{w}}}{M_{\mathrm{w}} C_{\mathrm{w}}}\left(\left(U_{\mathrm{bca}} A_{\mathrm{b}}\right)+\frac{h_{2} A_{\mathrm{b}} A_{\mathrm{g}} h_{\mathrm{kg}}\left(1-C_{1}\right)}{\left(h_{\mathrm{kg}} A_{\mathrm{g}}\right)+\left(h_{2} A_{\mathrm{b}}\right)}\right) \\
T_{\mathrm{b}}= & \frac{\alpha_{\mathrm{b}} I(t)+h_{\mathrm{bw}} T_{\mathrm{w}}+h_{\mathrm{wa}} T_{\mathrm{a}}}{h_{\mathrm{bw}}+h_{\mathrm{ba}}} \\
T_{\mathrm{go}}= & T_{\mathrm{w}}\left[\frac{(U A)_{\mathrm{wgo}}}{h_{1} A_{\mathrm{g}}+(U A)_{\mathrm{wgo}}}+\frac{h_{1} A_{\mathrm{g}}}{h_{1} A_{\mathrm{g}}+(U A)_{\mathrm{wgo}}}\right. \\
& \left.\times \frac{(U A)_{\mathrm{w}, \mathrm{wf}}}{(U A)_{\mathrm{w}, \mathrm{wf}}+h_{\mathrm{o}}}\left(1-\frac{1-\exp \left(-a_{2} l\right)}{a_{2} l}\right)\right] \\
& +\left(\frac{(I t)_{\mathrm{A}}}{h_{1} A_{\mathrm{g}}+(U A)_{\mathrm{wgo}}}+\frac{h_{1} A_{\mathrm{g}}}{h_{1} A_{\mathrm{g}}+(U A)_{\mathrm{wgo}}} S\right)
\end{aligned}
$$

$$
\begin{aligned}
T_{\mathrm{gi}}= & \frac{\alpha_{\mathrm{g}} I(t) A_{\mathrm{g}}}{h_{\mathrm{kg}} A_{\mathrm{g}}+h_{2} A_{\mathrm{b}}} \\
& +T_{\mathrm{w}}\left[\frac{\left(h_{2} A_{\mathrm{b}}\right)+\left(h_{\mathrm{kg}} A_{\mathrm{g}} C_{1}\right)}{h_{\mathrm{kg}} A_{\mathrm{g}}+h_{2} A_{\mathrm{b}}}\right] \\
& +\left[\frac{h_{\mathrm{kg}} A_{\mathrm{g}}}{h_{\mathrm{kg}} A_{\mathrm{g}}+h_{2} A_{\mathrm{b}}}\left\{\frac{h_{1} A_{\mathrm{g}}}{h_{1} A_{\mathrm{g}}+(U A)_{\mathrm{wgo}}} S+\frac{(I t)_{A}}{h_{1} A_{\mathrm{g}}+(U A)_{\text {wgo }}}\right\}\right]
\end{aligned}
$$$$
C_{1}=\frac{(U A)_{\mathrm{wgo}}}{h_{1} A_{\mathrm{g}}+(U A)_{\mathrm{wgo}}}
$$

$$
+\left(\frac{h_{1} A_{\mathrm{g}}}{h_{1} A_{\mathrm{g}}+(U A)_{\mathrm{wgo}}} \frac{(U A)_{\mathrm{w}, \mathrm{wf}}}{(U A)_{\mathrm{w}, \mathrm{wf}}+h_{o}}\left(1-\frac{1-\exp \left(-a_{2} l\right)}{a_{2} l}\right)\right)
$$

$S=\frac{\left((I t)_{3}+h_{\mathrm{o}} T_{\mathrm{a}}\right)}{\left(U A_{\mathrm{wwf}}+h_{\mathrm{o}}\right)}\left(1-\frac{1-\exp \left(-a_{2} l\right)}{a_{2} l}\right)+T_{\mathrm{wfi}}\left(\frac{1-\exp \left(-a_{2} l\right)}{a_{2} l}\right)$.

Unknown terms are expressed in "Appendix" 
Electrical efficiency $\left(\eta_{\mathrm{cN}}\right)$ of semitransparent photovoltaic module at $N$ th collector for Type (a) and Type (b) can be obtained from the following equation:

$$
N u=\frac{h_{\mathrm{cw}} \times d_{\mathrm{f}}}{k_{\mathrm{f}}}=C(G r \cdot P r)^{n}
$$

$\eta_{\mathrm{cN}}=\frac{\eta_{\mathrm{o}}\left[1-\beta_{\mathrm{o}}\left[\frac{1}{U_{\mathrm{tcp}}+U_{\mathrm{tca}}}\left[\left(I_{\mathrm{b}} Z_{\mathrm{a}}\right)+T_{\mathrm{a}}\left[U_{\mathrm{tca}}+\frac{U_{\mathrm{tp}} U_{\mathrm{L} 1}}{U_{\mathrm{L} 2}+\left(F^{\prime} h_{\mathrm{pf}}\right)}+\left(T_{\mathrm{a} 1} T_{\mathrm{a} 2}\right)\right]+T_{\mathrm{fi}} Z_{\mathrm{b}}\right]-T_{\mathrm{o}}\right]\right]}{1-\frac{\eta_{\mathrm{o}} \beta_{\mathrm{o}} I_{\mathrm{b}}}{\left(U_{\mathrm{tcp}}+U_{\mathrm{tca}}\right)}\left[Z_{\mathrm{c}}\right]}$.

Similarly, water temperature Eq. 16 for case (II) and case (III) can be obtained by substituting Type (b) and Type (c) design configurations in Eq. 8. The hourly yield, thermal energy $\left(E_{\text {thermal }}\right)$ and thermal exergy $\left(E x_{\text {thermal }}\right)$ for the cases (I-III) can be obtained from

$\dot{m}_{\mathrm{ew}}=\frac{h_{\mathrm{ew}} A_{\mathrm{b}}\left(T_{\mathrm{w}}-T_{\mathrm{gi}}\right)}{L} \times 3600$

and

$E x_{\text {thermal }}=h_{\mathrm{ew}} A_{\mathrm{b}}\left[\left(T_{\mathrm{w}}-T_{\mathrm{gi}}\right)-\left(T_{\mathrm{a}}+273\right) \times \ln \frac{T_{\mathrm{w}}+273}{T_{\mathrm{gi}}+273}\right]$,

where latent heat of vaporization $\left(\frac{\mathrm{kJ}}{\mathrm{kg}}\right)$ can be evaluated by Fernandez and Chargoy [30]

$$
\begin{aligned}
L= & 3.1625 \times 10^{6}+\left[1-\left(7.616 \times 10^{-4} \times T_{\mathrm{w}}\right)\right] \quad \text { if } T_{w}>70^{\circ} \mathrm{C} \\
L= & 2.4935 \times 10^{6}\left[1-\left(9.4779 \times T_{\mathrm{w}}\right)+\left(1.3132 \times 10^{-7} \times T_{\mathrm{w}}^{2}\right)\right. \\
& \left.-\left(4.7974 \times 10^{-3} \times T_{\mathrm{w}}^{3}\right)\right] \quad \text { if } T_{\mathrm{w}}<70^{\circ} \mathrm{C} .
\end{aligned}
$$

\section{Thermal model for heat transfer}

The temperature gradient in the fluid causes density variation in the humid air, which leads buoyant force and convective heat transfer in the solar still. Kumar and Tiwari [31] developed a thermal model based on regression method to determine convective $\left(h_{\mathrm{cw}}\right)$ and evaporative heat transfer coefficient $\left(h_{\mathrm{ew}}\right)$. They have considered the effects of solar still cavity, orientation of condensing cover (glass), operating temperature range during the thermal modeling, and assumed $100 \%$ relative humidity inside the solar still. This model can be used for the wider range of water temperature. The methodology carried out by Kumar and Tiwari [31] for the calculation of convective and evaporative heat transfer coefficient is as follows.

The rate of convective heat transfer from the water surface to the glass cover can be estimated by

$\dot{q}_{\mathrm{cw}}=h_{\mathrm{cw}}\left(T_{\mathrm{w}}-T_{\mathrm{gi}}\right) A_{\mathrm{b}}$,

where $h_{\mathrm{cw}}$ can be calculated from or

$h_{\mathrm{cw}}=\frac{C(G r \cdot P r)^{n} k_{\mathrm{f}}}{d_{\mathrm{f}}}$,

where

$G r=\frac{g \beta^{\prime} \rho^{2}\left(d_{\mathrm{f}}\right)^{3}(\Delta T)^{\prime}}{\mu^{2}}$

$\operatorname{Pr}=\frac{\mu C_{\mathrm{p}}}{k_{\mathrm{f}}}$

and

$\Delta T=\left(T_{\mathrm{w}}-T_{\mathrm{gi}}\right)+\left[\frac{\left(P_{\mathrm{w}}-P_{\mathrm{gi}}\right)\left(T_{\mathrm{w}}+273\right)}{\left(268.9 \times 10^{3}\right)-P_{\mathrm{w}}}\right]$

From Eq. (23c) it is observed that ' $h$ cw' depends upon ' $C$ ' and ' $n$ '. It was observed from different values of ' $C$ ' and ' $n$ ' for a particular range of Grashof number given by authors that the percentage deviation between experimental and theoretical is within reasonable percentage of accuracy for indoor simulated conditions; however, for outdoor conditions the deviation increases significantly. Thats why, Kumar and Tiwari [31] have modified the values of ' $C$ ' and ' $n$ ' for outdoor conditions.

The distillate output from an evaporative area $\left(A_{\mathrm{b}}\right)$ during time ' $t$ ' can be expressed as

$\dot{m}_{\mathrm{ew}}=\frac{\dot{q}_{\mathrm{ew}} \times t}{L}$,

where

$\dot{q}_{\mathrm{ew}}=A_{\mathrm{b}} h_{\mathrm{ew}}\left(T_{\mathrm{w}}-T_{\mathrm{gi}}\right)$

and

$h_{\mathrm{ew}}=16 \times 10^{-3} \times h_{\mathrm{cw}} \times \frac{\left(P_{\mathrm{w}}-P_{\mathrm{gi}}\right)}{\left(T_{\mathrm{w}}-T_{\mathrm{gi}}\right)}$.

Substituting, $h_{\mathrm{cw}}$ from Eq. (23c) in Eq. (23f)

$h_{\mathrm{ew}}=0.0162 \times C(G r \cdot P r)^{n} \times \frac{k_{\mathrm{f}}}{d_{\mathrm{f}}} \frac{\left(P_{\mathrm{w}}-P_{\mathrm{gi}}\right)}{\left(T_{\mathrm{w}}-T_{\mathrm{gi}}\right)}$. 
Further, substituting Eq. (23g) in Eq. (23e)

$\dot{q}_{\mathrm{ew}}=A_{\mathrm{b}} \times 0.0165 \times C(G r . P r)^{n} \times \frac{k_{\mathrm{f}}}{d_{\mathrm{f}}} \times\left(P_{\mathrm{w}}-P_{\mathrm{gi}}\right)$

Substituting Eq. (23h) in Eq. (23d), we get

$\dot{m}_{\mathrm{ew}}=K_{1} \times C(G r . P r)^{n}$

$\frac{\dot{m}_{\mathrm{ew}}}{K_{1}}=C(G r . P r)^{n}$,

where

$K_{1}=\frac{0.0162}{L} \times \frac{k_{\mathrm{f}}}{d_{\mathrm{f}}} \times A_{\mathrm{b}} \times t \times\left(P_{\mathrm{w}}-P_{\mathrm{gi}}\right)$.

Taking 'natural log' on both the sides of Eq. (23i) and comparing with $y=m x+C_{\mathrm{o}}$, we get

$\ln \left(\frac{\dot{m}_{\mathrm{ew}}}{K_{1}}\right)=\ln C+n \ln (G r . P r)$,

where

$y=\ln \left(\frac{\dot{m}_{\mathrm{ew}}}{K_{1}}\right)$

$C_{\mathrm{o}}=\ln C$

$x=\ln (G r . P r)$

$m=n$,

where $m=\frac{N\left(\sum_{i=1}^{N} x_{i} y_{i}\right)-\left(\sum_{i=1}^{N} x_{i}\right)\left(\sum_{i=1}^{N} y_{i}\right)}{N\left(\sum_{i=1}^{N} x_{i}^{2}\right)-\left(\sum_{i=1}^{N} x_{i}\right)^{2}}$

$C_{\mathrm{o}}=\frac{\left(\sum_{i=1}^{N} y_{i}\right)\left(\sum_{i=1}^{N} x_{i}^{2}\right)-\left(\sum_{i=1}^{N} x_{i}\right)\left(\sum_{i=1}^{N} x_{i} y_{i}\right)}{N\left(\sum_{i=1}^{N} x_{i}^{2}\right)-\left(\sum_{i=1}^{N} x_{i}\right)^{2}}$.

After calculating ' $m$ ' and ' $C_{\mathrm{o}}$ '

$C=\exp \left(C_{\mathrm{o}}\right)$

and, $n=m$

Thus, convective and evaporative heat transfer coefficients can be calculated with the help of these constants $(C, n)$ by substituting in Eq. (23c) and (23g).

The values of ' $C$ ', ' $n$ ', and Grahof number on the experimental day $(20 / 2 / 2018)$ at $8 \mathrm{~cm}$ of water depth in the proposed hybrid active solar still are $2.79,0.16$, and $2.8 \times 10^{-7}$ [31]. With these values, the convective $\left(h_{\mathrm{cw}}\right)$ and evaporative heat transfer $\left(h_{\text {ew }}\right)$ are $2.5 \mathrm{~W} / \mathrm{m}^{2}$ and $35 \mathrm{~W} / \mathrm{m}^{2}$.

\section{Regression analysis}

To find the relation between experimental value (Table 4) and theoretical value (Table 4 ) of yield, correlation coefficient $(r)$ and percentage deviation $(e)$ are calculated where

$r=\frac{N \sum_{i=1}^{N} X_{i} Y_{i}-\left(\sum_{i=1}^{N} X_{i} \sum_{i=1}^{N} Y_{i}\right)}{\sqrt{N \sum_{i=1}^{N} X_{i}^{2}-\left(\sum_{i=1}^{N} X_{i}\right)^{2}} \sqrt{N \sum_{i=1}^{N} Y_{i}^{2}-\left(\sum_{i=1}^{N} Y_{i}\right)^{2}}}$

$e=\sqrt{\frac{\sum_{i=1}^{N}\left(\frac{X_{i}-Y_{i}}{X_{i}}\right)^{2}}{N}} \times 100$.

The coefficient of correlation and percentage deviation of PVT-FPC SS-SS is 0.99 and $4.86 \%$ for the month of February' 2018.

\section{Performance parameters}

Performance analysis of three active solar distillation system cases (I-III) have been evaluated on the bases of the first and the second laws of thermodynamics; following Jafarkazemi and Ahmadifard [32], Nag [33].

1. The overall thermal energy and exergy analysis

$\dot{E}_{\text {daily, overall thermal }}=\sum_{t=1}^{24}\left[\dot{m}_{\mathrm{ew}} L\right]+\frac{\sum_{t=1}^{10}\left[\left[N A_{\mathrm{am}} I(t)_{\mathrm{b}}\left(\alpha \tau_{\mathrm{g}} \eta_{\mathrm{cN}} \rho\right)\right]-\dot{P}_{\mathrm{u}}\right]}{0.38}$

$\dot{E} x_{\text {daily, overall thermal }}=\sum_{t=1}^{24} h_{\mathrm{ew}} A_{\mathrm{b}}$

$\left[\left(T_{\mathrm{w}}-T_{\mathrm{gi}}\right)-\left(T_{\mathrm{a}}+273\right) \times \ln \frac{T_{\mathrm{w}}+273}{T_{\mathrm{gi}}+273}\right]$

$+\sum_{t=1}^{10}\left[\left[N A_{\mathrm{am}} I(t)_{\mathrm{b}}\left(\alpha \tau_{\mathrm{g}} \eta_{\mathrm{c} N} \rho\right)\right]-\dot{P}_{\mathrm{u}}\right]$

Collector exergy

$=\sum_{t=1}^{10}\left(\dot{m}_{\mathrm{f}} C_{\mathrm{f}}\right)\left[\left(T_{\mathrm{fo} N}-T_{\mathrm{fi}}\right)-\left(\left(T_{\mathrm{a}}+273\right) \times \ln \frac{T_{\mathrm{fo} N}+273}{T_{\mathrm{fi}}+273}\right)\right]$,

where $T_{\mathrm{fo} N}, T_{\mathrm{fi}}$, and $\dot{P}_{\mathrm{u}}$ represent the outlet fluid temperature at $N$ th PVT - CPC, inlet fluid temperature for the first PVT-CPC collector, and power consumed by the DC motor hourly. Thus, the daily thermal energy, the overall thermal energy, the overall thermal exergy, and 
Table 4 Hourly variations of various parameters of the hybrid active solar still for $0.08 \mathrm{~m}$ water depth on 20th February, 2018: (a) experimental value and (b) theoretical value

\begin{tabular}{|c|c|c|c|c|c|c|c|c|c|c|c|c|}
\hline Time & $I(t)_{\mathrm{C}}\left(\frac{\mathrm{W}}{\mathrm{m}^{2}}\right)$ & $I(t)_{\mathrm{S}}\left(\frac{\mathrm{W}}{\mathrm{m}^{2}}\right)$ & $T_{\mathrm{a}}\left({ }^{\circ} \mathrm{C}\right)$ & $V_{\mathrm{a}}\left({ }^{\circ} \mathrm{C}\right)$ & $V_{\mathrm{L}}(V)$ & $I_{\mathrm{L}}(A)$ & $V_{\mathrm{OC}}(V)$ & $I_{\mathrm{SC}}(A)$ & $T_{\mathrm{w}}\left({ }^{\circ} \mathrm{C}\right)$ & $T_{\mathrm{gi}}\left({ }^{\circ} \mathrm{C}\right)$ & $T_{\mathrm{b}}\left({ }^{\circ} \mathrm{C}\right)$ & $\dot{m}_{\mathrm{ew}}\left(\frac{\mathrm{kg}}{\mathrm{h}}\right)$ \\
\hline \multicolumn{13}{|c|}{ (a) Experimental value } \\
\hline 08:00 & 70 & 70 & 12 & 1.2 & 0 & 0 & 0 & 0 & 14.58 & 13.34 & 14.72 & 0.0043 \\
\hline 09:00 & 130 & 130 & 12 & 2 & 13.4 & 2.2 & 20.9 & 2.4 & 18.37 & 14.55 & 18.61 & 0.0091 \\
\hline $10: 00$ & 330 & 330 & 16.5 & 1.05 & 14.3 & 2.7 & 20.2 & 4.2 & 29.96 & 23.74 & 30.60 & 0.0252 \\
\hline $11: 00$ & 430 & 430 & 20 & 0.25 & 14.7 & 2.5 & 19.5 & 5.9 & 43.03 & 35.94 & 43.80 & 0.0609 \\
\hline $12: 00$ & 470 & 470 & 23.5 & 0.5 & 16.9 & 3.3 & 19 & 7.6 & 53.99 & 45.89 & 54.78 & 0.143 \\
\hline 13:00 & 510 & 510 & 24.5 & 1.75 & 16.5 & 2.8 & 19.2 & 7.5 & 61.29 & 50.58 & 62.11 & 0.3393 \\
\hline $14: 00$ & 490 & 490 & 25.5 & 1.25 & 16.6 & 2.7 & 18.5 & 7.2 & 65.95 & 57.60 & 66.68 & 0.3951 \\
\hline $15: 00$ & 450 & 450 & 27 & 1 & 15.6 & 2.6 & 18.3 & 5.2 & 68.26 & 61.29 & 68.89 & 0.4038 \\
\hline $16: 00$ & 350 & 350 & 26 & 0.55 & 13.5 & 2.5 & 17.6 & 4.3 & 66.33 & 60.83 & 66.73 & 0.3456 \\
\hline $17: 00$ & 210 & 210 & 25.5 & 0.9 & 13.1 & 2.3 & 16.2 & 3.5 & 59.30 & 53.40 & 59.43 & 0.3199 \\
\hline $18: 00$ & 70 & 70 & 23.5 & 0.45 & 0 & 0 & 0 & 0 & 55.87 & 49.70 & 55.52 & 0.2396 \\
\hline $19: 00$ & 50 & 50 & 20.5 & 0.55 & 0 & 0 & 0 & 0 & 52.45 & 45.48 & 52.11 & 0.2298 \\
\hline 20:00 & 0 & 0 & 18.5 & 0.45 & 0 & 0 & 0 & 0 & 49.31 & 42.25 & 48.9 & 0.1996 \\
\hline $21: 00$ & 0 & 0 & 17.1 & 0.55 & 0 & 0 & 0 & 0 & 46.31 & 38.76 & 46.0 & 0.1821 \\
\hline $22: 00$ & 0 & 0 & 15.9 & 0.05 & 0 & 0 & 0 & 0 & 43.86 & 37.52 & 43.57 & 0.1324 \\
\hline $23: 00$ & 0 & 0 & 14.3 & 0.45 & 0 & 0 & 0 & 0 & 41.27 & 33.48 & 40.98 & 0.1391 \\
\hline $24: 00$ & 0 & 0 & 14.3 & 0.55 & 0 & 0 & 0 & 0 & 38.88 & 31.21 & 38.62 & 0.1236 \\
\hline 01:00 & 0 & 0 & 13.5 & 1.05 & 0 & 0 & 0 & 0 & 36.46 & 27.80 & 36.21 & 0.1226 \\
\hline 02:00 & 0 & 0 & 13 & 1.05 & 0 & 0 & 0 & 0 & 34.26 & 25.91 & 34.035 & 0.1055 \\
\hline 03:00 & 0 & 0 & 13 & 1.05 & 0 & 0 & 0 & 0 & 32.30 & 24.40 & 32.09 & 0.0884 \\
\hline 04:00 & 0 & 0 & 12.5 & 1.05 & 0 & 0 & 0 & 0 & 30.51 & 22.86 & 30.32 & 0.0764 \\
\hline 05:00 & 0 & 0 & 12.5 & 1.05 & 0 & 0 & 0 & 0 & 28.91 & 21.72 & 28.73 & 0.0648 \\
\hline 06:00 & 0 & 0 & 11.5 & 1.05 & 0 & 0 & 0 & 0 & 27.38 & 20.22 & 27.21 & 0.0585 \\
\hline 07:00 & 0 & 0 & 11.5 & 1.05 & 0 & 0 & 0 & 0 & 26.00 & 19.31 & 25.85 & 0.0502 \\
\hline \multicolumn{13}{|c|}{ (b) Theoretical value } \\
\hline 08:00 & 70 & 70 & 12 & 1.2 & 0 & 0 & 0 & 0 & 14.67 & 13.38 & 14.85 & 0.0045 \\
\hline 09:00 & 130 & 130 & 12 & 2 & 13.4 & 2.2 & 20.9 & 2.4 & 18.60 & 14.64 & 18.92 & 0.0096 \\
\hline $10: 00$ & 330 & 330 & 16.5 & 1.05 & 14.3 & 2.7 & 20.2 & 4.2 & 30.54 & 24.04 & 31.37 & 0.0269 \\
\hline $11: 00$ & 430 & 430 & 20 & 0.25 & 14.7 & 2.5 & 19.5 & 5.9 & 43.97 & 36.64 & 44.99 & 0.0655 \\
\hline $12: 00$ & 470 & 470 & 23.5 & 0.5 & 16.9 & 3.3 & 19 & 7.6 & 55.21 & 46.95 & 56.26 & 0.154 \\
\hline 13:00 & 510 & 510 & 24.5 & 1.75 & 16.5 & 2.8 & 19.2 & 7.5 & 62.67 & 51.90 & 63.78 & 0.3643 \\
\hline $14: 00$ & 490 & 490 & 25.5 & 1.25 & 16.6 & 2.7 & 18.5 & 7.2 & 67.42 & 59.11 & 68.42 & 0.4217 \\
\hline $15: 00$ & 450 & 450 & 27 & 1 & 15.6 & 2.6 & 18.3 & 5.2 & 69.74 & 62.85 & 70.62 & 0.4295 \\
\hline $16: 00$ & 350 & 350 & 26 & 0.55 & 13.5 & 2.5 & 17.6 & 4.3 & 67.71 & 62.29 & 68.30 & 0.3659 \\
\hline $17: 00$ & 210 & 210 & 25.5 & 0.9 & 13.1 & 2.3 & 16.2 & 3.5 & 60.42 & 54.58 & 60.68 & 0.3384 \\
\hline $18: 00$ & 70 & 70 & 23.5 & 0.45 & 0 & 0 & 0 & 0 & 56.87 & 50.73 & 56.52 & 0.2522 \\
\hline $19: 00$ & 50 & 50 & 20.5 & 0.55 & 0 & 0 & 0 & 0 & 53.34 & 46.38 & 52.99 & 0.2412 \\
\hline 20:00 & 0 & 0 & 18.5 & 0.45 & 0 & 0 & 0 & 0 & 50.10 & 43.04 & 49.76 & 0.2088 \\
\hline $21: 00$ & 0 & 0 & 17.1 & 0.55 & 0 & 0 & 0 & 0 & 47.01 & 39.44 & 46.69 & 0.1901 \\
\hline $22: 00$ & 0 & 0 & 15.9 & 0.05 & 0 & 0 & 0 & 0 & 44.50 & 38.14 & 44.19 & 0.1378 \\
\hline $23: 00$ & 0 & 0 & 14.3 & 0.45 & 0 & 0 & 0 & 0 & 41.84 & 34.01 & 41.54 & 0.1446 \\
\hline $24: 00$ & 0 & 0 & 14.3 & 0.55 & 0 & 0 & 0 & 0 & 39.39 & 31.66 & 39.12 & 0.1284 \\
\hline 01:00 & 0 & 0 & 13.5 & 1.05 & 0 & 0 & 0 & 0 & 36.91 & 28.17 & 36.66 & 0.1272 \\
\hline 02:00 & 0 & 0 & 13 & 1.05 & 0 & 0 & 0 & 0 & 34.65 & 26.23 & 34.42 & 0.1092 \\
\hline 03:00 & 0 & 0 & 13 & 1.05 & 0 & 0 & 0 & 0 & 32.65 & 24.67 & 32.45 & 0.0913 \\
\hline 04:00 & 0 & 0 & 12.5 & 1.05 & 0 & 0 & 0 & 0 & 30.83 & 23.09 & 30.63 & 0.0788 \\
\hline 05:00 & 0 & 0 & 12.5 & 1.05 & 0 & 0 & 0 & 0 & 29.19 & 21.92 & 29.01 & 0.0668 \\
\hline
\end{tabular}


Table 4 (continued)

\begin{tabular}{ccccccccccccc}
\hline Time & $I(t)_{\mathrm{C}}\left(\frac{\mathrm{w}}{\mathrm{m}^{2}}\right)$ & $I(t)_{\mathrm{S}}\left(\frac{\mathrm{w}}{\mathrm{m}^{2}}\right)$ & $T_{\mathrm{a}}\left({ }^{\circ} \mathrm{C}\right)$ & $V_{\mathrm{a}}\left({ }^{\circ} \mathrm{C}\right)$ & $V_{\mathrm{L}}(V)$ & $I_{\mathrm{L}}(A)$ & $V_{\mathrm{OC}}(V)$ & $I_{\mathrm{SC}}(A)$ & $T_{\mathrm{w}}\left({ }^{\circ} \mathrm{C}\right)$ & $T_{\mathrm{gi}}\left({ }^{\circ} \mathrm{C}\right)$ & $T_{\mathrm{b}}\left({ }^{\circ} \mathrm{C}\right)$ & $\left(\frac{\mathrm{kg}}{\mathrm{h}}\right)$ \\
\hline $06: 00$ & 0 & 0 & 11.5 & 1.05 & 0 & 0 & 0 & 0 & 27.63 & 20.40 & 27.46 & 0.0602 \\
$07: 00$ & 0 & 0 & 11.5 & 1.05 & 0 & 0 & 0 & 0 & 26.23 & 19.47 & 26.08 & 0.0516 \\
\hline
\end{tabular}

Table 5 Optimized parameters for Type $(\mathrm{a}-\mathrm{c})$ for the months of June and January

\begin{tabular}{|c|c|c|c|c|c|c|}
\hline S. no. & Cases & $\begin{array}{l}\text { Mass flow rate } \\
\text { (CC) }(\mathrm{kg} / \mathrm{s})\end{array}$ & $\begin{array}{l}\text { Heat } \\
\text { exchanger } \\
\text { (m) }\end{array}$ & $\begin{array}{l}\text { Mass of } \\
\text { water }(\mathrm{kg})\end{array}$ & $\begin{array}{l}\text { No. of col- } \\
\text { lectors }\end{array}$ & $\begin{array}{l}\text { Mass flow rate } \\
\text { (kg/s) (collec- } \\
\text { tor) }\end{array}$ \\
\hline \multirow[t]{3}{*}{1.} & Type (a) & & & & & \\
\hline & June & 0.025 & 1.97 & 280 & 6 & 0.04 \\
\hline & January & 0.025 & & 280 & 7 & 0.04 \\
\hline \multirow[t]{3}{*}{2.} & Type (b) & & & & & \\
\hline & June & 0.025 & 1.97 & 280 & 8 & 0.04 \\
\hline & January & 0.025 & & 250 & 10 & 0.04 \\
\hline \multirow[t]{3}{*}{3.} & Type (c) & & & & & \\
\hline & June & 0.025 & 1.97 & 280 & 5 & 0.04 \\
\hline & January & 0.025 & & 280 & 6 & 0.04 \\
\hline
\end{tabular}

Table 6 Daily energy and exergy for the months of January and June for the three cases (I-III)

\begin{tabular}{|c|c|c|c|c|c|c|c|}
\hline \multirow[t]{2}{*}{ S. no. } & \multirow[t]{2}{*}{ Parameters } & \multicolumn{2}{|l|}{ Case (I) } & \multicolumn{2}{|l|}{ Case (II) } & \multicolumn{2}{|l|}{ Case (III) } \\
\hline & & June (kWh) & January (kWh) & June $(\mathrm{kWh})$ & January (kWh) & June (kWh) & January (kWh) \\
\hline 1. & Thermal energy & 27.08 & 24.08 & 16.86 & 11.36 & 28.59 & 26.56 \\
\hline 2. & Overall thermal energy & 29.75 & 28.81 & 33.64 & 39.84 & 28.06 & 26.07 \\
\hline 3. & Overall exergy & 2.82 & 3.87 & 7.00 & 11.44 & 1.86 & 2.59 \\
\hline 4. & Collector exergy & 1.62 & 3.21 & - & - & 1.87 & 3.70 \\
\hline
\end{tabular}

Table 7 Energy payback time, energy production factor, and life cycle conversion efficiency on the basis of energy and exergy for the three cases (I-III)

\begin{tabular}{lllll}
\hline S. no. & Parameters & Case (I) & Case (II) & Case (III) \\
\hline 1. & Total embodied energy $(\mathrm{kWh})$ & 9556.34 & $18,209.50$ & 7023.50 \\
2. & Annual yield $(\mathrm{kg})$ & 8126.48 & 5451.28 & 8743.48 \\
3. & Annual overall thermal energy available from SS-SS (kWh) & 6347.30 & 7776.48 & 6123.25 \\
4. & Annual overall thermal exergy available from SS-SS (kWh) & 591.22 & 1715.89 & 345.86 \\
5. & Energy payback time based on energy (years) & $1.50-2$ & 2.34 & $1.14-2$ \\
6. & Energy payback time based on exergy (years) & 17 & 10.61 & 20 \\
7. & Energy production factor based on energy & 0.66 & 0.42 & 0.87 \\
8. & Energy production factor based on exergy & 0.06 & 0.09 & 0.04 \\
9. & Life & 50 & 50 & 50 \\
10. & Solar radiation for life time (kWh) & $9,77,464$ & $12,00,753$ & $8,47,541$ \\
11. & Life cycle conversion efficiency based on energy & 0.31 & 0.30 & 0.35 \\
12. & Life cycle conversion efficiency based on exergy & 0.022 & 0.06 & 0.002 \\
\hline
\end{tabular}

the collector exergy have been evaluated and are represented in Table 6. Here, it should be noted that after sunshine hours, yield $\left(\dot{m}_{\text {ew }}\right)$ is continuously obtained from the active solar stills [case (I-III)] because water mass in the basin acts as a thermal storage.

2. Economic analysis
The evaluation of energy matrices is an important tool for the renewable energy technologies (RES) to be successful. Energy matrices include the study of (1) energy payback time (EPBT), (2) energy production factor (EPF), and (3) life cycle conversion efficiency (LCCE) of the system, Tiwari and Mishra [34]. Table 7 
Table 8 Embodied energy of the three hybrid distillation systems case (I-III)

\begin{tabular}{lllll}
\hline S. no. & Name of components & \multicolumn{3}{l}{ Embodied energy (kWh) } \\
\cline { 3 - 5 } \cline { 3 - 4 } & & Case (I) & Case (II) & Case (III) \\
\hline 1. & Single slope solar still & 1737.79 & 1737.7 & 1737.7 \\
2. & Heat exchanger & 350.5 & 350.5 & 350.5 \\
3. & Compound parabolic concentrator collector & 5733 & 7371 & 4914 \\
4. & Photovoltaic module (glass to glass) & 1715 & 8731.8 & 0 \\
5. & Others & 20.00 & 20.00 & 20.00 \\
\multicolumn{2}{l}{ Total embodied energy (kWh) } & 9556.34 & $18,209.50$ & 7023.50 \\
\hline
\end{tabular}

Table 9 Capital investment for the three hybrid solar distillation systems [case (IIII)]
Table 10 Uniform end-of-year annual cost for the three hybrid solar distillation systems case (I-III)

\begin{tabular}{llrrr}
\hline S. no. & Cost of components & \multicolumn{3}{l}{ Cost of system (₹) } \\
\cline { 3 - 5 } & & Case (I) & Case (II) & Case (III) \\
\hline 1. & Single slope solar still & 23,143 & 23,143 & 23,143 \\
2. & Photovoltaic thermal-compound parabolic concentrator @ 10,500 & 73,500 & 0 & 0 \\
3. & Photovoltaic module-compound parabolic concentrator @ 17,750 & 0 & $1,59,750$ & 0 \\
4. & Flat plate collector-compound parabolic concentrator @9250 & 0 & 0 & 55,500 \\
5. & Heat exchanger & 5100 & 5100 & 5100 \\
6. & Motor/pump & 1000 & 1000 & 1000 \\
7. & Fabrication & 6000 & 6000 & 6000 \\
8. & Total capital investment & $1,08,743$ & $1,94,993$ & 90,743
\end{tabular}

\begin{tabular}{llrrlrlll}
\hline S. no. & $n$ (years) & $i(\%)$ & \multicolumn{1}{c}{$P_{\mathrm{s}}(\mathrm{Rs})$} & $M @ 10 \%$ & \multicolumn{1}{c}{$S_{\mathrm{s}}(\mathrm{Rs})$} & $F_{\mathrm{CR}, \mathrm{i}, \mathrm{n}}$ & $S_{\mathrm{SR}, \mathrm{i}, \mathrm{n}}$ & UAC (₹) \\
\hline Case (I) & & & & & & & & \\
1. & 50 & 2 & $1,08,743$ & 10,874 & 29,251 & 0.031 & 0.01 & 3386 \\
2. & 50 & 5 & $1,08,743$ & 10,874 & $1,24,699$ & 0.054 & 0.004 & 5960 \\
3. & 50 & 10 & $1,08,743$ & 10,874 & $12,76,543$ & 0.1 & 0.00085 & 10,876 \\
Case (II) & & & & & & & & \\
4. & 50 & 2 & $1,94,993$ & 19,499 & 52,484 & 0.031 & 0.01 & 6071 \\
5. & 50 & 5 & $1,94,993$ & 19,499 & $2,23,606$ & 0.054 & 0.004 & 10,688 \\
6. & 50 & 10 & $1,94,993$ & 19,499 & $22,89,039$ & 0.1 & 0.00085 & 19,503 \\
Case (III) & & & & & & & & \\
7. & 50 & 2 & 90,743 & 9074 & 24,424 & 0.031 & 0.01 & 2825.6 \\
8. & 50 & 5 & 90,743 & 9074 & $1,04,058$ & 0.054 & 0.004 & 4973.8 \\
9. & 50 & 10 & 90,743 & 9074 & $10,65,239$ & 0.1 & 0.00085 & 9076.2 \\
\hline
\end{tabular}

represents energy matrices of the cases (I-III) based on energy and exergy.

3. Production cost of distilled water (₹ $/ \mathrm{kg})$ and electricity generation (₹/kWh)

Initially, total embodied energy (kWh) (Table 8) for the three hybrid solar distillation systems cases (I-III) is calculated. Then, the capital investment for the three hybrid solar distillation systems is calculated from Eq. 27 (Table 9). In the cost analysis, replacement period of pump/motor is considered to be 10, 20, and 30 years, respectively. Following Tiwari and Mishra [34], a mathematical expression for capital recovery factor $\left(F_{\mathrm{CR}, \mathrm{i}, \mathrm{n}}\right)$, sinking fund factor $\left(S_{\mathrm{CR}, \mathrm{i}, \mathrm{n}}\right)$, and uniform end-of-year annual cost (UAC) is calculated in Table 10. Following Kumar and Tiwari [35], production cost of distilled water $\left(C_{\mathrm{wp}}\right)(₹ / \mathrm{kg})$ and electricity generation $\left(C_{\mathrm{e}}\right)(₹ /$ $\mathrm{kWh}$ ) is calculated (Table 11) from Eq. 27

$$
\begin{gathered}
P=P_{\mathrm{SS}_{-} \mathrm{SS}}+P_{\mathrm{HE}}+P_{\mathrm{CPC}_{-} \text {collector }}+P_{\mathrm{PVM}}+P_{\text {fabrication }} \\
\mathrm{UAC}=\left(P_{\mathrm{s}} \times F_{\mathrm{CR}, \text { in }}\right)+\left(M_{\mathrm{s}} \times F_{\mathrm{CR}, \text { in }}\right)-\left(S_{\mathrm{S}} \times F_{\mathrm{SR}, \text { in }}\right) ; \\
C_{\mathrm{wp}}=\frac{\mathrm{UAC}-R_{\mathrm{e}}}{M_{\mathrm{w}}} ; \quad C_{\mathrm{e}}=\frac{\mathrm{UAC}-R_{\mathrm{w}}}{E_{\mathrm{e}}} .
\end{gathered}
$$


Table 11 Production cost of distilled water obtained from the three cases (I-III)

\begin{tabular}{|c|c|c|c|c|c|c|c|c|c|c|c|c|}
\hline S. no. & $i(\%)$ & $\begin{array}{l}\text { UAC } \\
\text { (Rs) }\end{array}$ & $M_{\mathrm{w}}(\mathrm{kg})$ & $E_{\mathrm{e}}(\mathrm{kWh})$ & $\begin{array}{l}(\mathrm{SP})_{\mathrm{w}}(₹ / \\
\mathrm{kg})\end{array}$ & $\begin{array}{l}(\mathrm{SP})_{\mathrm{e}} \\
(\mathrm{₹} / \mathrm{kWh})\end{array}$ & $R_{\mathrm{w}}(₹)$ & $\operatorname{Re}(₹)$ & $\begin{array}{l}\mathrm{UAC}-R_{\mathrm{e}} \\
\text { (₹) }\end{array}$ & $\begin{array}{l}\mathrm{UAC}-R_{\mathrm{w}} \\
\text { (₹) }\end{array}$ & $\begin{array}{l}C_{\mathrm{wp}} \\
(\mathrm{₹} / \mathrm{kg})\end{array}$ & $\begin{array}{l}C_{\mathrm{e}} \\
\text { (₹/kWh) }\end{array}$ \\
\hline \multicolumn{13}{|c|}{ Case (I) } \\
\hline 1. & 2 & 3386 & 8126 & 490 & 5 & 5 & 40,632 & 2450 & 936 & 0 & 0.11 & 0 \\
\hline 2. & 5 & 5960 & 8126 & 490 & 5 & 5 & 40,632 & 2450 & 3510 & 0 & 0.43 & 0 \\
\hline 3. & 10 & 10,876 & 8126 & 490 & 5 & 5 & 40,632 & 2450 & 8426 & 0 & 1.03 & 0 \\
\hline \multicolumn{13}{|c|}{ Case (II) } \\
\hline 4. & 2 & 6071 & 5451 & 1582 & 5 & 5 & 27,255 & 7910 & 0 & 0 & 0 & 0 \\
\hline 5. & 5 & 10,688 & 5451 & 1258 & 5 & 5 & 27,255 & 7910 & 2778 & 0 & 0.50 & 0 \\
\hline 6 & 10 & 19,503 & 5451 & 1582 & 5 & 5 & 27,255 & 7910 & 11,593 & 0 & 2.12 & 0 \\
\hline \multicolumn{13}{|c|}{ Case (III) } \\
\hline 7 & 2 & 2825.6 & 8743 & 0 & 5 & 5 & 43,717 & 0 & 2825.6 & 0 & 0.32 & 0 \\
\hline 8 & 5 & 4973.8 & 8743 & 0 & 5 & 5 & 43,717 & 0 & 4973.8 & 0 & 0.56 & 0 \\
\hline 9 & 10 & 9076.2 & 8743 & 0 & 5 & 5 & 43,717 & 0 & 9076.2 & 0 & 1.03 & 0 \\
\hline
\end{tabular}

Table 12 Co-generation efficiency for the hybrid solar distillation system [case (I-II)]

\begin{tabular}{lll}
\hline S. no. & Cases & $\begin{array}{l}\text { Co-generation } \\
\text { efficiency (\%) }\end{array}$ \\
\hline 1. & Case (I) & 44 \\
2. & Case (II) & 24 \\
\hline
\end{tabular}

Here, $R_{\mathrm{w}}, R_{\mathrm{e}}$, and UAC represent the revenue earned from water and electricity, and uniform end-of-year annual cost obtained from the three cases (I-III). If $\mathrm{UAC}-R_{\mathrm{e}}$ or $\mathrm{UAC}-R_{\mathrm{w}}$ gives negative term, it is considered to be zero, which means the revenue obtained from the other source is capable to overcome the total cost of the system.
4. Co-generationefficiency

Co-generation is the simultaneous generation of electricity $(\mathrm{kWh})$ and thermal energy $(\mathrm{kWh})$. The production of electricity in conventional power plant releases heat (thermal energy), which is discarded as waste, whereas in co-generation this thermal energy is utilized for heating. From case (I) and case (II), electricity (power) is generated by glass-to-glass photovoltaic module and thermal energy (yield) simultaneously, due to solar energy (Table 12). Here, the electrical energy obtained is the net difference between electricity generated and consumed by the DC motor. Following Onovwiona and Ugursal [36], co-generation efficiency of the case (I) and (III) can be expressed as follows:

$\eta_{\operatorname{cog}}=\frac{\text { Electrical energy }+ \text { thermal energy }}{\text { Solar radiation input to the system }}$.
Fig. 2 Hourly variation of global, beam solar radiation, and ambient temperature for the months of January and June, respectively

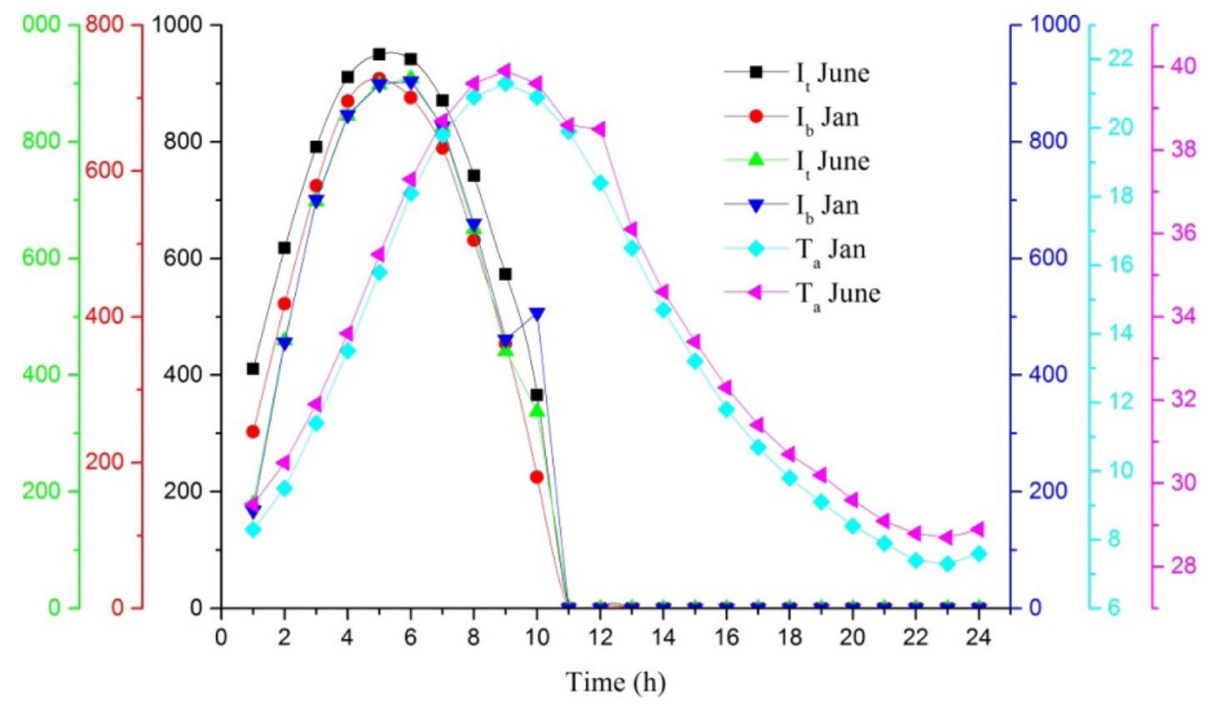




\section{Methodology}

The following methodology is carried out for the study of three hybrid distillation systems:

Step (i) Initially following Lui and Jordan formulae [37], beam radiation $\left(I_{\mathrm{b}}\right)($ Online resource 1$)$ for PVT-CPC kept an angle of $30^{\circ}$ from the horizontal and global radiation $(I(t))$ (Online resource 1 ) for the condensing cover inclined at $15^{\circ}$ on SS-SS facing southwards is calculated for the months of January and June (Fig. 2). Further annual calculations of $I_{\mathrm{b}}$ (Online resource 1) and $I(t)$ (Online resource 1) is carried out simultaneously by the summation of solar intensity for the different months, which is done by multiplying daily solar radiation with number of clear days, hazy days, hazy and cloudy days, and cloudy days for a given month.

Step (ii) Optimizing the parameters for maximizing outlet fluid temperature $\left(T_{\mathrm{fo} N}\right)$ (Eq. 8), module efficiency $\left(\eta_{\mathrm{m}}\right)$ (Eq. 20) and useful gain $\left(\dot{Q}_{\mathrm{u}, N}\right)$ (Eq. 11) have been carried out for three Type $(\mathrm{a}-\mathrm{c})$ on hourly, daily, and monthly bases. On the basis of numerical computation, optimized parameters are given in Table 5.

Step (iii) Further, water temperature $\left(T_{\mathrm{w}}\right)$, basin temperature $\left(T_{\mathrm{b}}\right)$, inner glass temperature $\left(T_{\mathrm{gi}}\right)$, and hourly yield $\left(\dot{m}_{\text {ew }}\right)$ are calculated hourly and daily using Eqs. 16, 17, 19, and 21. Then, performance parameters, energy matrices, embodied energy $(\mathrm{kWh})$, production cost of distilled water $\left(C_{\mathrm{wp}}\right)$ and electricity $\left(C_{\mathrm{e}}\right)$, and co-generation efficiency are obtained from Eqs. 24-28.

Thereafter, cases (I-III) are compared on the bases of computed numerical values.

\section{Numerical computation}

\section{Case (A) Optimization of the number of thermal collectors, length of heat exchanger, mass flow rate in PVT-CPC collector loop, and above condensing cover}

Optimization of the three active distillation system parameters assists for the maximum yield $\left(\dot{m}_{\text {ew }}\right)$, exergy $\left(\mathrm{Ex}_{\text {thermal }}\right)$, and lowers energy back time (EPBT) and production cost of distilled water and electricity $\left(C_{\mathrm{wp}}\right.$ and $\left.C_{\mathrm{e}}\right)$. Figure $3 \mathrm{a}-\mathrm{c}$ shows that at the lower mass flow rate $\left(\dot{m}_{\mathrm{f}}\right)$ higher outlet fluid temperature $\left(T_{\mathrm{foN}}\right)$ (Eq. 8$)$ is obtained for the month of June for all three Type $(\mathrm{a}-\mathrm{c})$. At higher mass flow rate, the curves for the months of January and June intersect each other at the high number of thermal collectors $(N)$. This is because thermal losses are maximum at higher operating temperature for the month of June. That's why, after four thermal collectors [case (I)] the outlet fluid temperature $\left(T_{\mathrm{foN}}\right)$ for the month of January dominates. A similar effect is observed by Singh and Tiwari [28]. Thus, the optimization of mass flow rate for thermal collectors [Type $(a-c)$ ]
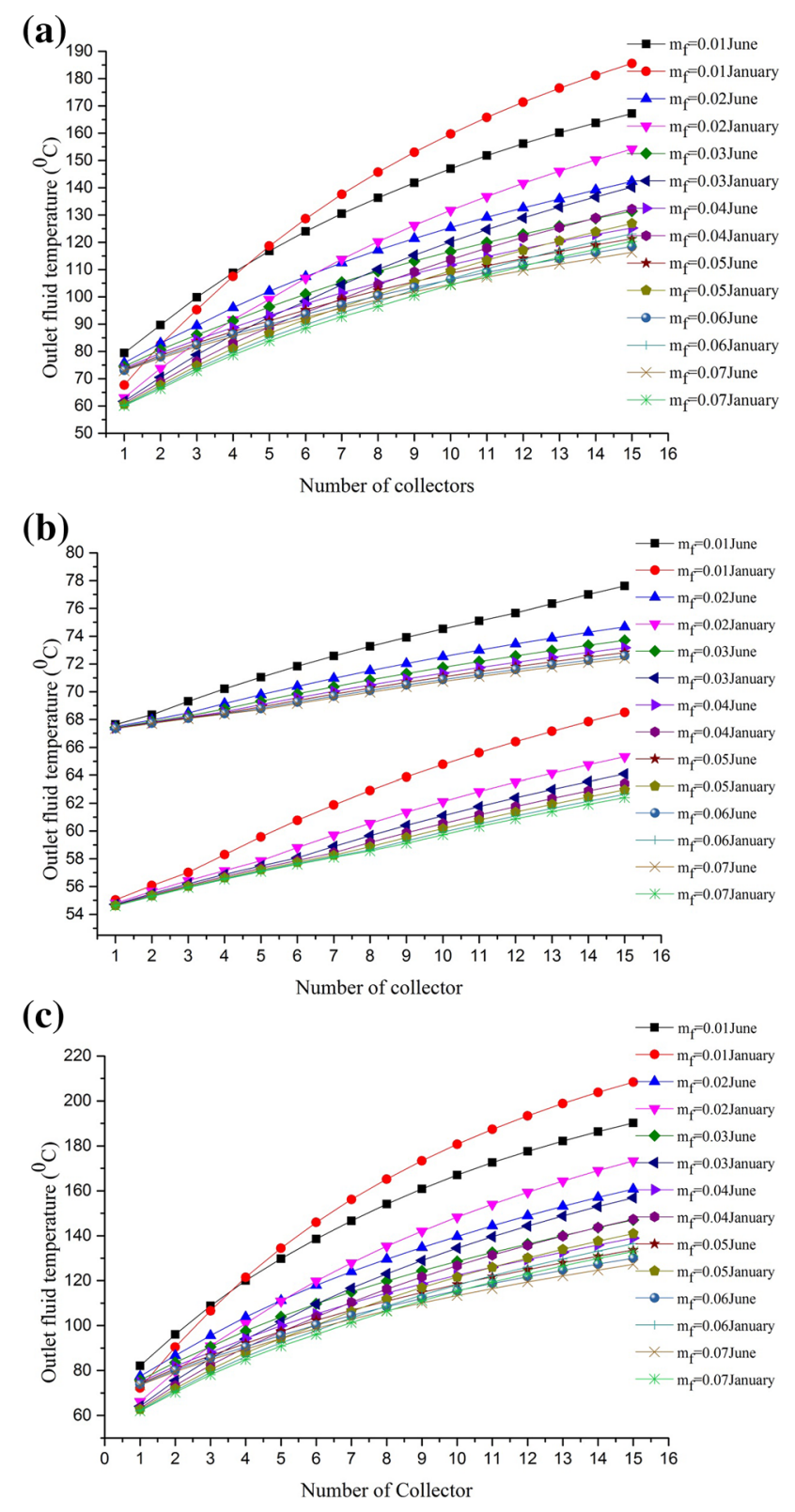

Fig. 3 a Variation of outlet fluid temperature with the number of collectors at a given mass flow rate for case (I) for the months of January and June, respectively. b Variation of outlet fluid temperature with the number of collectors at a given mass flow rate for case (II) for the months of January and June, respectively. c Variation of outlet fluid temperature with the number of collectors at a given mass flow rate for case (III) for the months of January and June, respectively

and water flowing above condensing cover and number of thermal collectors for three Type $(\mathrm{a}-\mathrm{c})$ is carried out and represented in Table 5. Case (II) has lesser thermal losses as outlet fluid temperature $\left(T_{\text {fo } N}\right)$ reaches the maximum of 75 and $66^{\circ} \mathrm{C}$ for $0.01 \mathrm{~kg} / \mathrm{s}$ mass flow rate in the months of June and January, respectively. Similarly, for the case (III) 
Fig. 4 a Variation of inlet fluid temperature with the number of helix of heat exchanger for case (I) for the months of January and June, respectively. b Variation of inlet fluid temperature with the number of helix of heat exchanger for case (II) for the months of January and June, respectively. c Variation of inlet fluid temperature with the number of helix of heat exchanger for case (III) for the months of January and June, respectively
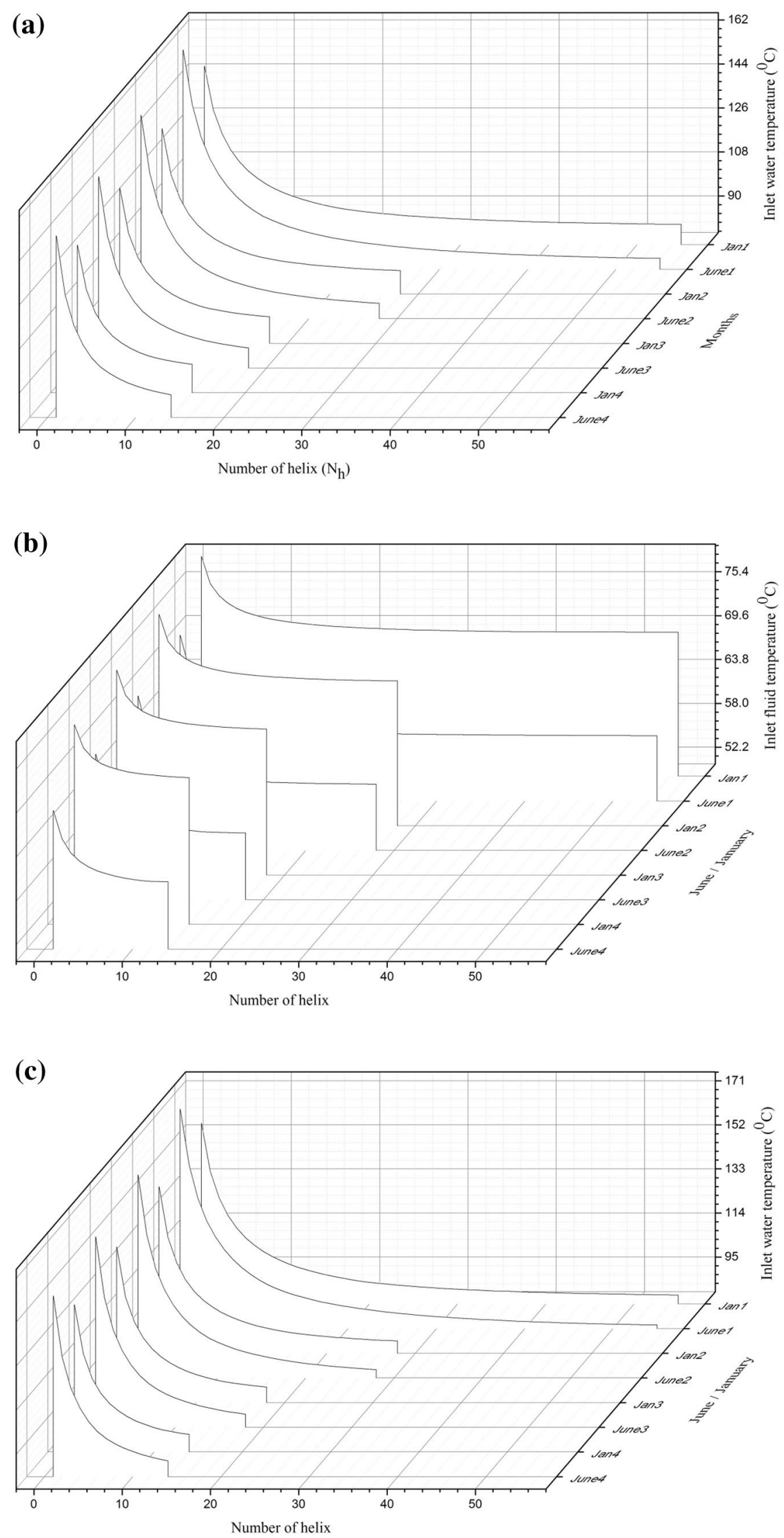


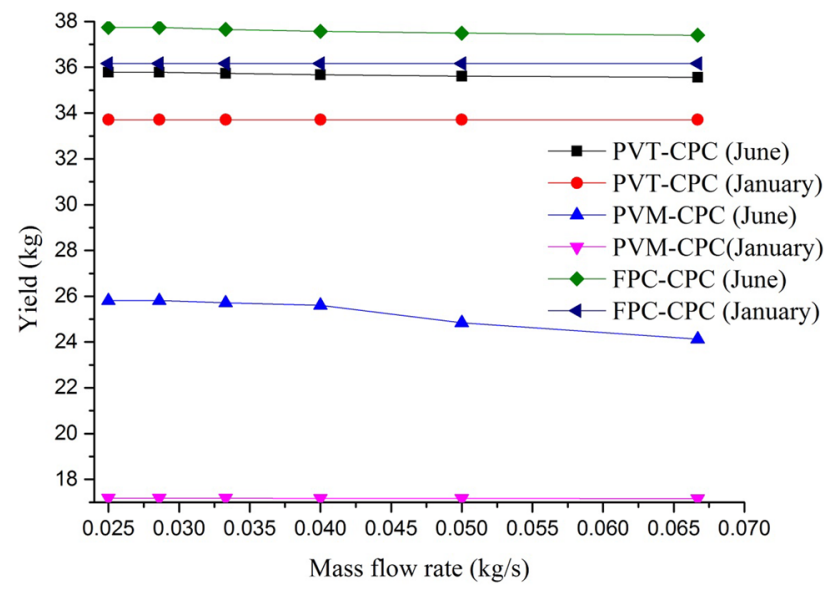

Fig. 5 Variation of yield with the mass flow rate flowing over condensing cover for three cases (I-III) for the months of January and June, respectively

after three thermal collectors the outlet fluid temperature $\left(T_{\text {foN }}\right)$ for the month of January dominates compared to the month of June at a mass flow rate of $0.01 \mathrm{~kg} / \mathrm{s}$. Now, the maximum thermal heat is transferred to the brackish water mass in the basin by the optimization for number of helix (14) and pitch $(0.05 \mathrm{~m})$ of heat exchanger (Fig. $4 \mathrm{a}-\mathrm{c}$ ) for the months of June and January, respectively. For maximizing the overall heat transfer coefficient $(U)$ from the heat exchanger of length $(L)$, different pitches ranging from $(0.0125$ to $0.05 \mathrm{~m})$ and helix $(55,35,28$ and 14$)$ are considered for the minimum inlet fluid temperature $\left(T_{\mathrm{fi}}\right)$. In Fig. $4 \mathrm{a}$, [case (I)] the minimum inlet water temperature is obtained for the months of June and January as 79 and $86^{\circ} \mathrm{C}$, respectively, for the configuration having 14 helix of $0.05 \mathrm{~m}$ pitch. Minimum inlet water temperature $\left(T_{\mathrm{fi}}\right)$ in the month of June clearly signifies that more heat transfer occurs from the fluid inside the heat exchanger (water) to the brackish water in the basin compared to January. Other configuration of the heat exchanger also follows the same behavior. Similarly, for the cases (II) and (III), the minimum inlet water temperature $\left(T_{\mathrm{fi}}\right)$ is $69,58{ }^{\circ} \mathrm{C}$ and $87,86^{\circ} \mathrm{C}$ (Fig. $4 \mathrm{~b}$, c) for the configuration of 14-helix and $0.05 \mathrm{~m}$ pitch. So, this configuration is optimized for maximum overall heat transfer, among others for the months of June and January, respectively, for three configurations cases (I-III). Water flowing above condensing cover has a significant role on the overall performance of the active solar distillation systems cases (i-iii). A uniform water mass flowing over the condensing cover $\left(\dot{m}_{\mathrm{f} 1}\right)$ remarkably increases the yield (distilled water) (Eq. 21) obtained from the solar still, as shown in Fig. 5. The reason behind it is that as the temperature difference between brackish water in the basin $\left(T_{\mathrm{w}}\right)$ and inner glass $\left(T_{\mathrm{gi}}\right)$ increases, the yield increases $\left(\dot{m}_{\mathrm{ew}}\right)$ (Eq. 21). The mass flow rate of water flowing over condensing cover is reduced from 0.065 to $0.025 \mathrm{~kg} / \mathrm{s}$ simultaneously with the decrease in temperature to maximize yield (energy) and exergy. One can observe in Fig. 5 that with the increase in mass flow rate over the condensing cover the yield decreases as the contact time period between water and condensing cover is less. Maximum yield of $37.9 \mathrm{~kg}$ for case (III) is obtained in the month of June and minimum $17.18 \mathrm{~kg}$ for case (II) for the month of January (Fig. 5), at the mass flow rate over condensing cover of $0.025 \mathrm{~kg} / \mathrm{s}$. Thus, the mass flow rate of $0.025 \mathrm{~kg} / \mathrm{s}$ is optimized for the three cases (I-III). Moreover, the effect of length of heat exchanger over the daily yield $(\mathrm{kg})$ with different mass flow rates $\left(\dot{m}_{\mathrm{f}}\right)(\mathrm{kg} / \mathrm{s})$ varying from 0.01 to $0.07 \mathrm{~kg} / \mathrm{s}$ for the optimized thermal collector (Table 5) is studied from Fig. 6a-c. The graphs show the results as expected, i.e., the daily yield increases with the length of heat exchanger with the mass flow rate varying from 0.01 to $0.07 \mathrm{~kg} / \mathrm{s}$ for all the cases (I-III) for the months of June and January, respectively. The reason is that as the length of heat exchanger increases from 0.5 to $1.97 \mathrm{~m}$ higher heat transfer occurs from fluid (inside the heat exchanger) to brackish water mass in the basin. The optimization of higher mass flow rate of water over condensing cover is less desirable as it lowers the exergy of the system. Therefore, a specific mass flow rate in thermal collector and over cooling condensing cover is studied and optimized for higher energy and exergy. At optimized conditions for the three active distillation systems cases (I-III), (Table 5) maximum yield obtained from case (I) is 35.9 and $34.1 \mathrm{~kg}$, case (II) is 25.8 and $17.2 \mathrm{~kg}$, and case (III) is 37.9 and $36.6 \mathrm{~kg}$ for the months of June and January, respectively.

The yield $\left(\dot{m}_{\text {ew }}\right)$ obtained from the proposed system case (I) (without heat exchanger $(L)$ ) for the month of June is 14.8\% higher than Singh and Tiwari [35]. Because the latent heat of condensation released after condensation of water vapor at the inner condensing cover is absorbed by the flowing water over the outer glass cover, which enhances the condensation and evaporation process. This results in enhancement of the yield (distilled water).

\section{Case (B) Hourly and daily performance analysis on the bases of energy and exergy}

On the bases of the above optimized parameters, hourly and daily performances for the three cases (I-III) have been studied. Daily yield (kg) (Eq. 21) and thermal exergy (kWh) (Eq. 22) for three cases (I-III) is calculated for the months of June and January, and the results are shown in Fig. 7. Case (III) shows maximum yield $37.9 \mathrm{~kg}$ and maximum exergy $2.54 \mathrm{kWh}$ for the month of June for a particular day. It was observed that the increase in yield simultaneously reduces exergy as the entropy (thermal losses) is generated. Thus, optimization of system parameters is done for maximizing energy and exergy. Case (I) gives $8 \%$ higher yield for the 
Fig. 6 a Variation of yield with length of heat exchanger at different mass flow rate for case (I) for the months of January and June, respectively. b Variation of yield with length of heat exchanger at different mass flow rate for case (II) for the months of January and June, respectively. c Variation of yield with length of heat exchanger at different mass flow rate for case (III) for the months of January and June, respectively
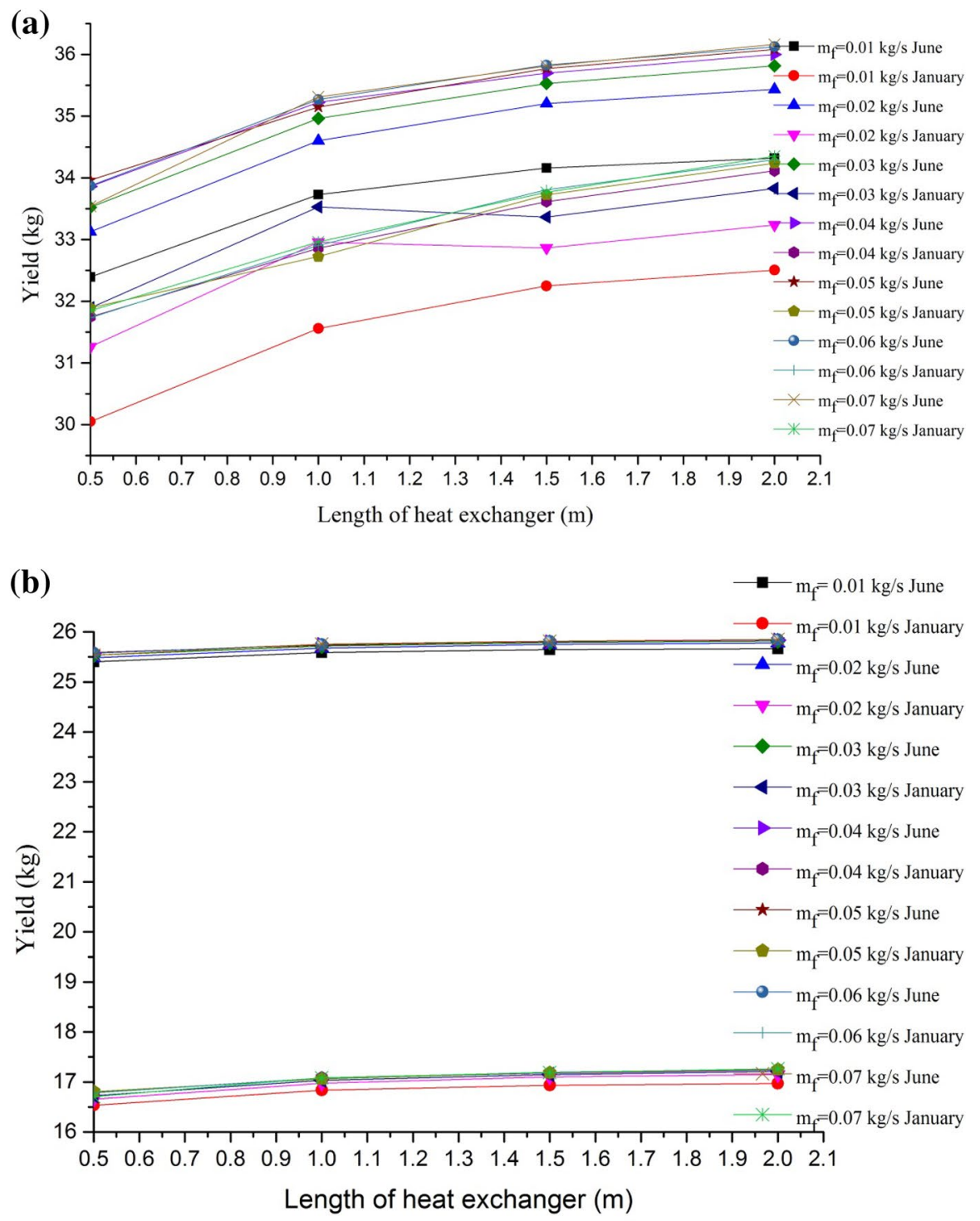

(c)

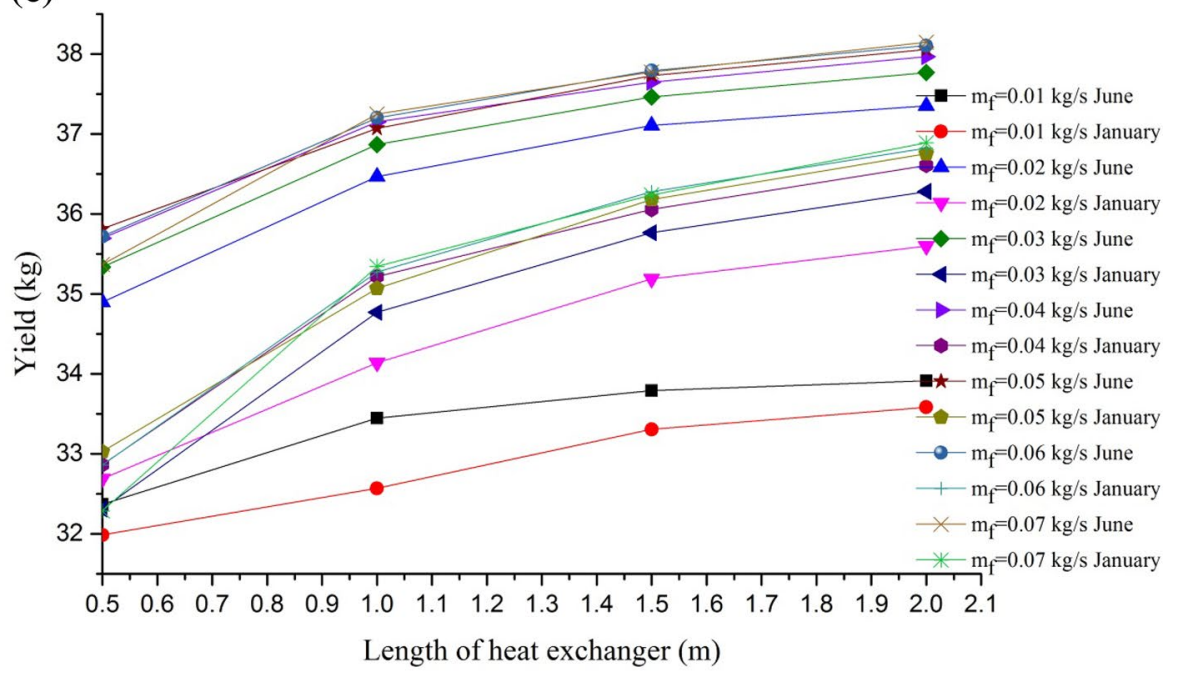




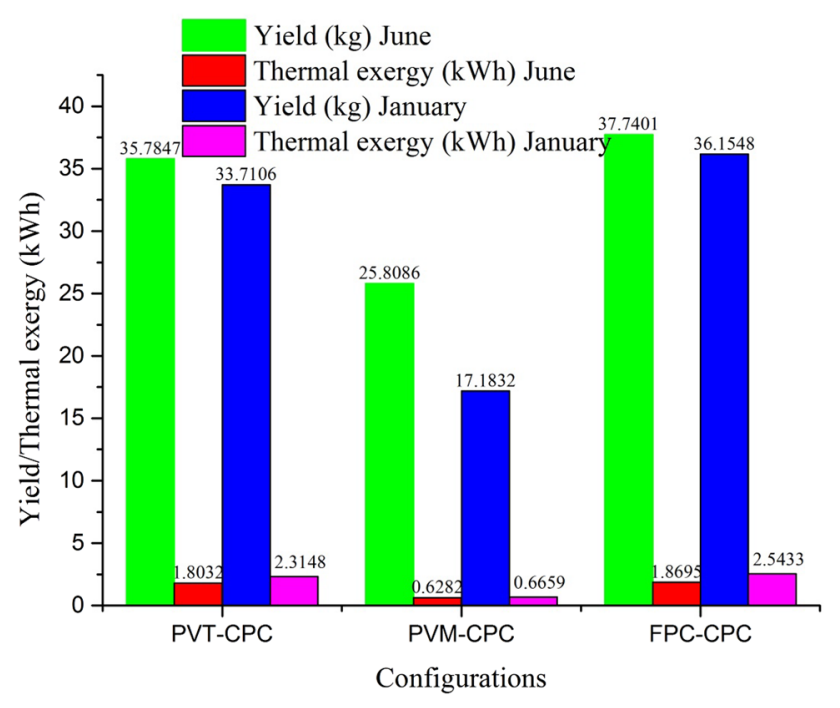

Fig. 7 Daily yield and thermal exergy for three cases (I-III) for the months of June and January, respectively

month of June compared to January and 36\% higher exergy in the month of January because of the above mentioned reason. Similarly, case (II) and case (III) give 37 and $4 \%$ higher yield and 18.5 and $68 \%$ higher exergy in the month of June and January, respectively.

For cases (I) and (II), DC electrical energy is generated from semitransparent photovoltaic module from which, approximately $20 \mathrm{~W}$ is consumed in DC motor and further remaining power can be commercialized. The electrical efficiency $\left(\eta_{\mathrm{m}}\right)$ (Eq. 20) of solar modules decreases when there is rise in temperature of solar cells; if the thermal energy generated by solar cells is extracted it will result in increased electrical efficiency of module and lowers the probability of degradation of solar cell by thermal heating. Former, behavior of solar photovoltaic modules is observed in Fig. 8, and the latter is observed in Fig. 9, where the case (II) gives highest electrical efficiency of $13 \%$ for the month of January corresponding to minimum solar cell $\left(\eta_{\mathrm{m}}\right)$ temperature of $53{ }^{\circ} \mathrm{C}$ because of cooling condensing cover. Similarly, minimum electrical efficiency is obtained from case (I) for the month of June is $11 \%$, corresponding to $75.69^{\circ} \mathrm{C}$ of solar cell temperature $\left(T_{\mathrm{c} N}\right)$. The increased electrical efficiency $\left(\eta_{\mathrm{m}}\right)$ analogous to lesser cell temperature $\left(T_{\mathrm{c} N}\right)$ is obtained because a constant water mass of $0.025 \mathrm{~kg} / \mathrm{s}$ flowing above the condensing cover $\left(T_{\mathrm{go}}\right)$ extracts the dissipated thermal heat from the outer glass cover $\left(T_{\mathrm{go}}\right)$, enhancing evaporation and simultaneously transfer of heat from the heat exchanger
Fig. 8 Hourly variation of cell temperature and module efficiency for case (I) and case (II) for the months of June and January, respectively
Fig. 9 Variation of cell temperature and module efficiency for case (I) and case (II) with mass flow rate flowing over the condensing cover for the months of June and January, respectively
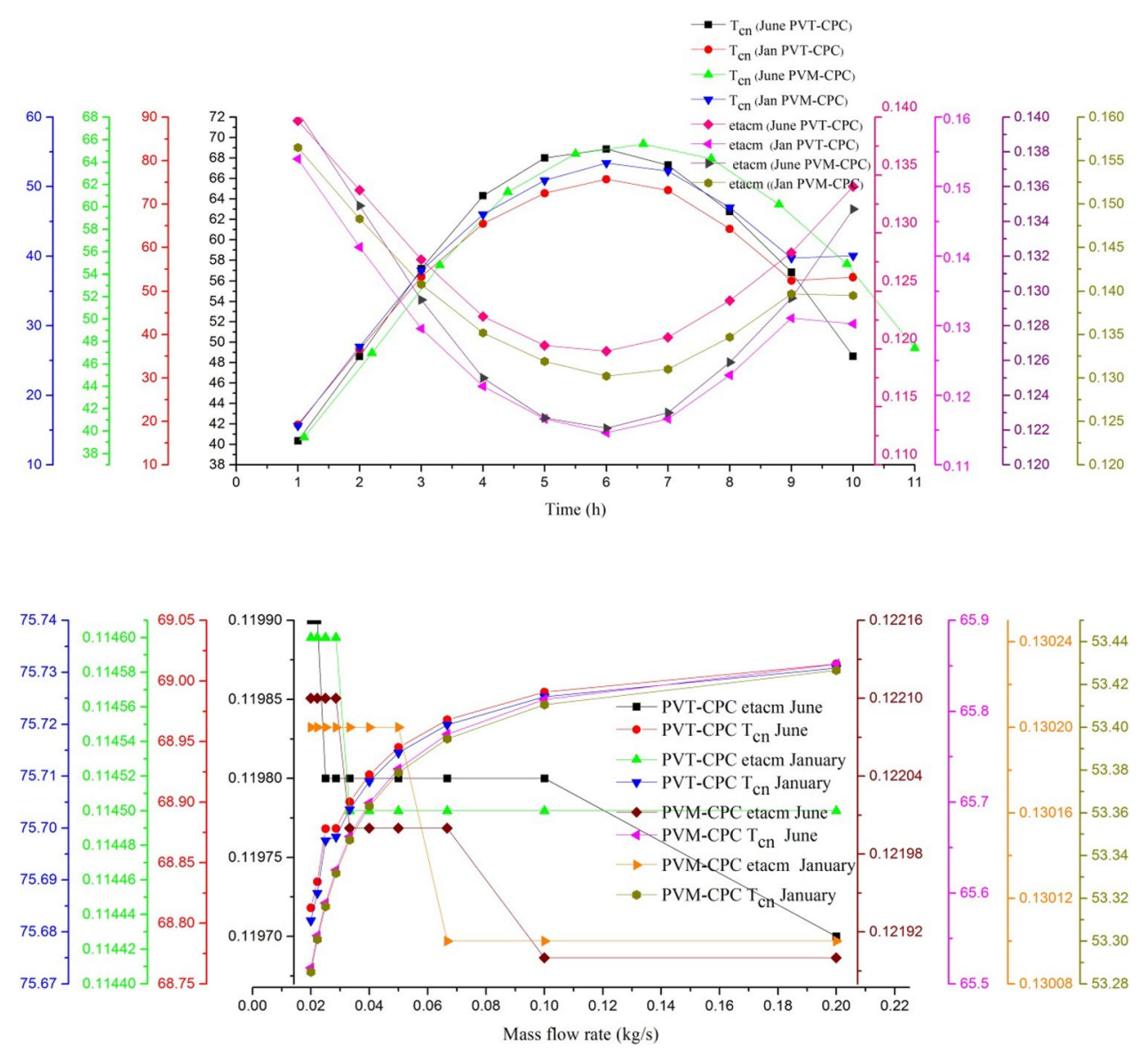


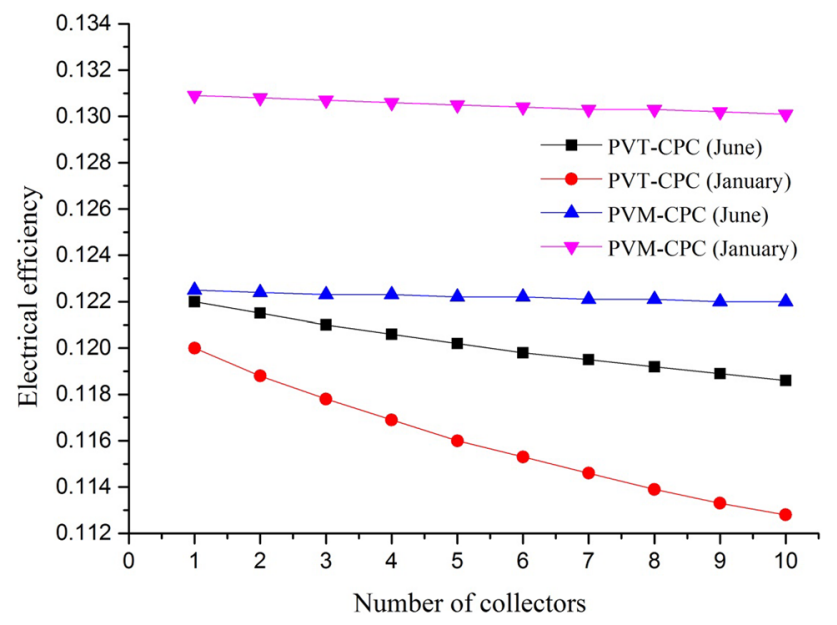

Fig. 10 Variation of electrical efficiency with number of collectors for case (I) and case (II) for the months of January and June, respectively

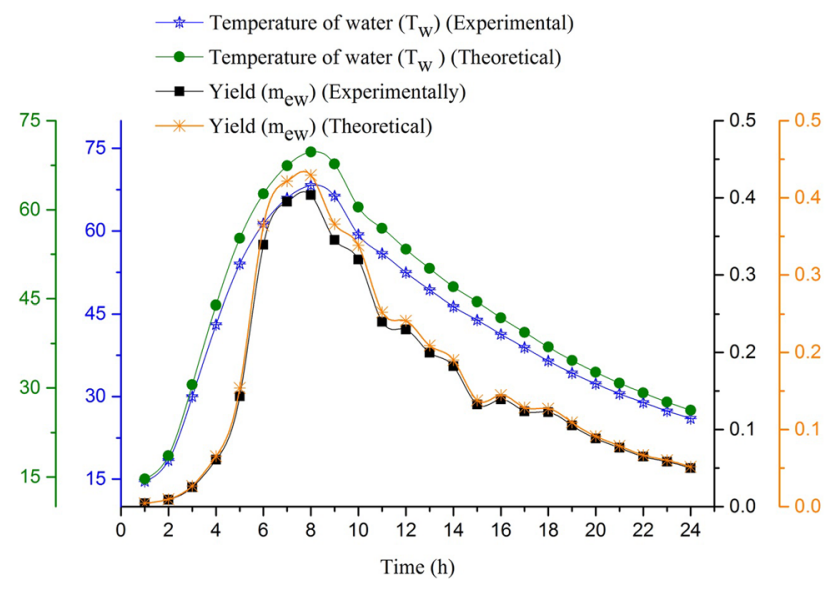

Fig. 11 Variation of water temperature and yield obtained from PVTFPC-SS-SS

to water mass in the basin, thus higher thermal energy is extracted from thermal collectors which comprise photovoltaic modules as well. As a result, photovoltaic modules are cooled, giving higher electrical efficiency. This effect occurs in two active solar distillation systems case (I) and case (II) (Fig. 9). The decreasing electrical efficiency $\left(\eta_{\mathrm{m}}\right)$ curve with increasing solar cell temperature $\left({ }^{\circ} \mathrm{C}\right)$ curve at higher mass flow rate of water $\left(\dot{m}_{\mathrm{f} 1}\right)$ also confirms the reason that at higher mass flow rate as the contact time period is less, which lowers module efficiency and yield. The numerical values obtained in Fig. 9 are similar to the values obtained in Fig. 8.

The decreasing electrical efficiency behavior with the increase in the number of collectors is represented in Fig. 10. The fluid (water) flowing beneath the first semitransparent photovoltaic in thermal collectors Type $(a, b)$ is at lower temperature thus, more electrical efficiency is obtained.

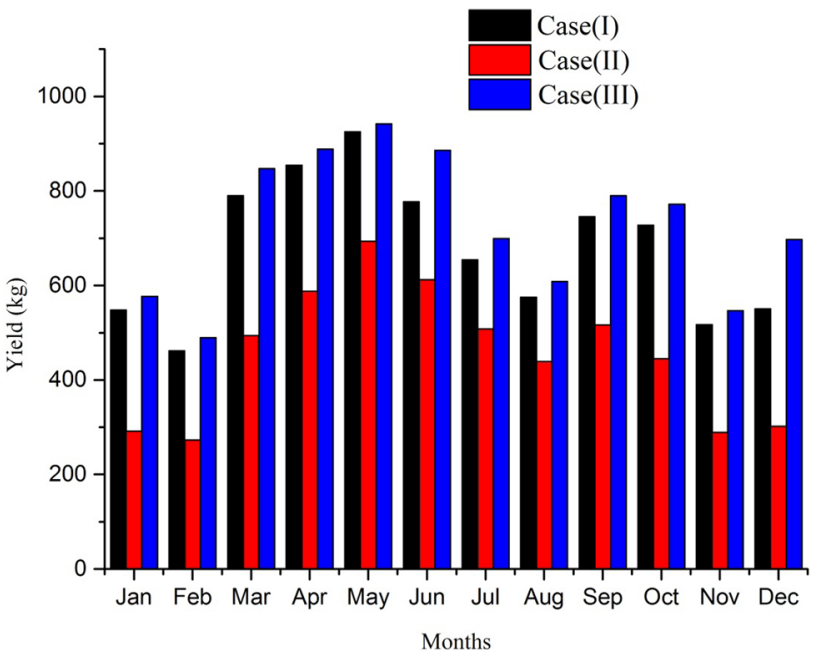

Fig. 12 Monthly variation of yield (distilled water) for three cases (IIII)

In consecutive thermal collectors, the fluid temperature is slightly higher than the previous ones, which lead to reduced electrical efficiency as higher temperature of water will extract lesser thermal energy liberated from solar cells. The maximum electrical efficiency obtained from Type (b) from single semitransparent photovoltaic module in the month of January is $13 \%$ and it decreases gradually with ten photovoltaic thermal collectors. Similar behavior is observed with Type (a) for the months of both June and January, respectively. Figure 11 shows the comparison of theoretical and experimental work done for the PVT-FPC-SS-SS (special case). The maximum temperature of water $\left(T_{\mathrm{w}}\right)$ obtained from theoretical/predicted work is $69.74{ }^{\circ} \mathrm{C}$ and from the experimental work is $68.26^{\circ} \mathrm{C}$. Similarly, yield is maximum around 3:00 p:m for both experimental and theoretical works, i.e., 0.4038 and $0.4295 \mathrm{~kg}$.

The comparison of daily thermal energy $(\mathrm{kWh})$, overall thermal energy $(\mathrm{kWh})$, overall exergy $(\mathrm{kWh})$, and collector exergy $(\mathrm{kWh})$ for the three cases (I-III) is represented in Table 6 for the months of June and January. Higher thermal energy ( $\mathrm{kWh}$ ) leads to higher yield thus, case (III) is $15.5 \%$, $10.2 \%$, and $57.6 \%, 133 \%$ higher in the month of June and January, respectively, in comparison to case (I) and case (II). The overall thermal energy (kWh) is highest for case (II), i.e., 39.84 as the electrical gain is higher because of 10 fully covered photovoltaic thermal collectors, for the month of January. A percentage increase of overall thermal energy in case (II) is $13 \%, 38 \%$ higher than case (I) and 19\%, 52\% higher than case (III) for the months of June and January, respectively. The overall thermal exergy ( $\mathrm{kWh}$ ) for case (II) is maximum, i.e., $11.44 \mathrm{kWh}$ and minimum for case (III), i.e., $1.86 \mathrm{kWh}$ for the months of January and June, respectively. Collector exergy calculated in case (I) and case (III) shows that exergy in case 
(III) is $15.43 \%, 15.26 \%$ higher from case (I) as expected, for the months of June and January, respectively.

\section{Case (C) Monthly performance analysis on the bases of energy and exergy}

Extending the values obtained from the daily basis to annual basis for the three cases (I-III), an annual yield (Fig. 12), overall thermal energy, overall thermal exergy, electrical energy, and solar radiation acts as input for the calculation of energy matrices, production cost of water and electricity, and co-generation efficiency. Energy matrices calculated on the bases of energy and exergy are represented in Table 7 for three active solar still cases (I-III). The energy payback time follows the order $(\text { EPBT })_{\text {case (III) }}<(\text { EPBT) })_{\text {case (I) }}<(\text { EPBT) })_{\text {case (II) }}$. Case (III) shows minimum energy payback time of 1 year because its embodied energy (kWh) is minimum (Table 8 ) and yield (Table 7) is maximum. The value of EPBT on the basis of exergy is lowest for case (II) (10 years) because higher electrical exergy is obtained from Type (b). Energy production factor (EPF) on the basis of energy is $57 \%$ higher in case (I) than case (II) and $24 \%$ lesser than case (III). Moreover, on the basis of exergy case (II) is 50\% higher than case (I) and $125 \%$ higher than case (III). Life cycle conversion efficiency (LCCE) is highest for case (III) on the basis of energy as embodied energy is 26\% lesser than case (I) and 61\% lesser than case (II). Similarly, on the basis of exergy, LCCE is highest for case (II).

\section{Case (D) Production cost of water and electricity, and co-generation efficiency for three cases (I-III)}

The calculation of the capital investment $(P)$, uniform endof-year annual cost (UAC), and production cost of water $\left(C_{\mathrm{wp}}\right)$ and electricity $\left(C_{\mathrm{e}}\right)($ Eq. 27$)$ for the three cases (I-III) is represented in Tables 9,10, and 11. Table 9 represents the cost of various components in (₹) utilized in the three hybrid solar distillation systems. Total capital investment of three cases (I-III) is ₹ 1,08,743, ₹ 1,94,993, and ₹ 90,743. Case (II) has a higher investment cost because of the higher number of fully covered photovoltaic thermal-compound parabolic concentrators (Table 5). The production cost of distilled water obtained from three cases (I-III) varies from Rs 0.11 to ₹ $2.12 / \mathrm{kg}$. Case (i) gives a minimum production cost of water, i.e., ₹ $0.11 / \mathrm{kg}$ at $2 \%$ rate of interest which is 78\% lesser than Singh and Tiwari [36] because the yield is higher and UAC is lower for the optimized parameters (Table 5) thus, system gets economically viable. The electricity generated in case (II), i.e., $1582 \mathrm{kWh}$, whereas distilled water obtained is $5451 \mathrm{~kg}$. Thus, the hybrid active solar still [cases (II)] is sustainable from distillation point of view as production cost of electricity $\left(C_{\mathrm{e}}\right)$ is null, which means the production cost of water $\left(C_{\mathrm{wp}}\right)$ itself is capable of overcoming the total cost (UAC) of the system. Case (III) gives a production cost of water 190\% higher than case (I) at a $2 \%$ rate of interest thus, it is less desirable. An interest rate of 5 or $10 \%$ gives the higher value of the production cost of water and electricity compared to $2 \%$. The co-generation efficiency for the two cases (I) and (II) is represented in Table 12 . The co-generation efficiency is $83 \%$ higher in case (I) compared to case (II) as electrical energy and thermal energy (yield) both are obtained in case (I), and the input solar radiation is $18 \%$ lesser for the life time of 50 years compared to case (II).

\section{Conclusions}

The following summarized conclusions have been drawn from the present study:

1. The optimum mass flow rates for the thermal collectors and water above condensing cover $\left(T_{\mathrm{go}}\right)$ are 0.04 and $0.025 \mathrm{~kg} / \mathrm{s}$ for $1.97 \mathrm{~m}$ length of heat exchanger and $280 \mathrm{~kg}$ mass of water in basin for maximum energy and exergy of the three active solar distillation systems. The optimized numbers of thermal collectors are 6,8 , and 5 for the month of June and 7, 10, and 6 for the month of January for the three cases, respectively.

2. On the bases of overall thermal energy, daily electrical efficiency, energy payback time, production cost of water and electricity, and co-generation efficiency case (I) gives better results than case (II) and case (III).

3. In case (III) thermal energy, overall thermal exergy and collector exergy is higher and capital investment, uniform annual end-of-year annual cost (UAC) is lower.

4. Case (I), with cooling condensing cover is proposed for domestic and industrial purposes over case (II) and case (III), as it fulfills the commercial purposes such as charging of batteries, cleaning of medical equipment, avoiding scaling in equipments, and giving higher distilled water and electrical energy (direct current).

Acknowledgements The authors acknowledge the Department of Science and Technology (DST), Indian Institute of Technology-Delhi (IITD) for the financial help during the research work. We would also like to express our gratitude to the researchers who helped in the preparation of this manuscript.

\section{Compliance with ethical standards}

Conflict of interest On behalf of all the authors, the corresponding author states that there is no conflict of interest.

Open Access This article is distributed under the terms of the Creative Commons Attribution 4.0 International License (http://creat ivecommons.org/licenses/by/4.0/), which permits unrestricted use, 
distribution, and reproduction in any medium, provided you give appropriate credit to the original author(s) and the source, provide a link to the Creative Commons license, and indicate if changes were made.

\section{Appendix}

$$
\begin{aligned}
& Z_{1}=\left(\alpha_{\mathrm{c}} \beta_{\mathrm{c}} \tau_{\mathrm{g}} \rho \frac{A_{\mathrm{am}}}{A_{\mathrm{rm}}}\right) ; \\
& Z_{2}=\frac{U_{\text {tcp }}}{\left(U_{\mathrm{L} 2}+\left(F^{\prime} h_{\mathrm{pf}}\right)\right)}\left\{(\alpha \tau)_{2, \mathrm{eff}}+P F_{1} \alpha_{\mathrm{c}} \beta_{\mathrm{c}} \tau_{\mathrm{g}} \rho \frac{A_{\mathrm{am}}}{A_{\mathrm{rm}}}\right\} \\
& Z_{3}=\frac{U_{\mathrm{tcp}}\left(\left(F^{\prime} h_{\mathrm{pf}}\right)+U_{\mathrm{tpf}}\right)}{2\left(\dot{m}_{\mathrm{f}} C_{\mathrm{f}}\left(U_{\mathrm{L} 2}+\left(F^{\prime} h_{\mathrm{pf}}\right)\right)\right)} \\
& Z_{4}=\left(P F_{2}(\alpha \tau)_{2, \mathrm{eff}}\right)+\left(P F_{1} P F_{2} \alpha_{\mathrm{c}} \beta_{\mathrm{c}} \tau_{\mathrm{g}} \rho \frac{A_{\mathrm{am}}}{A_{\mathrm{rm}}}\right) A_{\mathrm{rm}} F_{\mathrm{rm}} \\
& Z_{5}=\frac{U_{\mathrm{tcp}}\left(F^{\prime} h_{\mathrm{pf}}+U_{\mathrm{tpf}}\right)}{2\left(\dot{m}_{\mathrm{f}} C_{\mathrm{f}}\left(U_{\mathrm{L} 2}+\left(F^{\prime} h_{\mathrm{pf}}\right)\right)\right)} ; \quad Z_{6}=P F_{\mathrm{c}}\left(A_{\mathrm{rc}} F_{\mathrm{rc}}\right)(\alpha \tau)_{\mathrm{c}, \mathrm{eff}} \\
& Z_{7}=\left(P F_{2}(\alpha \tau)_{2, \mathrm{eff}}\right) \\
& +\left(P F_{2} P F_{1} \alpha_{\mathrm{c}} \tau_{\mathrm{g}} \beta_{\mathrm{c}} \rho \frac{A_{\mathrm{am}}}{A_{\mathrm{rm}}}\right) A_{r m} F_{r m}\left(1-\frac{A_{\mathrm{rc}} F_{\mathrm{rc}} U_{\mathrm{LC}}}{\dot{m}_{\mathrm{f}} C_{\mathrm{f}}}\right) \\
& Z_{8}=\left(1+K_{\mathrm{m}}\right) \frac{\left(1-K_{\mathrm{K}}^{N-1}\right)}{1-K_{\mathrm{K}}} \text {; } \\
& Z_{\mathrm{a}}=\left[(Z 1+Z 2)+\left(Z_{3} Z_{4}\right)+Z_{5}\left[\left\{Z_{6}+Z_{7}\right\} Z_{8}\right]\right] \\
& T_{\mathrm{a} 1}=\frac{U_{\mathrm{tcp}}\left(\left(F^{\prime} h_{\mathrm{pf}}\right)+U_{\mathrm{tpf}}\right)}{2 \dot{m}_{\mathrm{f}} C_{\mathrm{f}}\left(U_{L 2}+\left(F^{\prime} h_{\mathrm{pf}}\right)\right)} \\
& T_{\mathrm{a} 2}=\left(A F_{\mathrm{R}} U_{\mathrm{L}}\right)_{\mathrm{m} 1}+\left(\left(A F_{\mathrm{R}} U_{\mathrm{L}}\right)_{1} \frac{\left(1-K_{\mathrm{K}}^{N-1}\right)}{\left(1-K_{\mathrm{K}}\right)}\left(1+K_{\mathrm{m}}\right)\right) \\
& Z_{\mathrm{b}}=\left[\frac{U_{\mathrm{tcp}}\left(F^{\prime} h_{\mathrm{pf}}+U_{\mathrm{tpf}}\right)\left(1+K_{\mathrm{m}}\right) K_{\mathrm{K}}^{N-1}}{2\left(U_{\mathrm{L} 2}+F^{\prime} h_{\mathrm{pf}}\right)}\right] \text {; } \\
& Z_{9}=\tau_{\mathrm{g}} \beta_{\mathrm{c}} \rho \frac{A_{\mathrm{am}}}{A_{\mathrm{rm}}} ; \quad Z_{10}=\frac{U_{\mathrm{tcp}}}{\left(U_{\mathrm{L} 2}+\left(F^{\prime} h_{\mathrm{pf}}\right)\right)} P F_{1} \tau_{\mathrm{g}} \beta_{\mathrm{c}} \rho \frac{A_{\mathrm{am}}}{A_{\mathrm{rm}}} \\
& Z_{11}=\frac{U_{\mathrm{tcp}}\left(F^{\prime} h_{\mathrm{pf}}+U_{\mathrm{tpf}}\right)}{2 \dot{m}_{\mathrm{f}} C_{\mathrm{f}}\left(U_{\mathrm{L} 2}+F^{\prime} h_{\mathrm{pf}}\right)} P F_{1} P F_{2} \tau_{\mathrm{g}} \beta_{\mathrm{c}} \frac{A_{\mathrm{am}}}{A_{\mathrm{rm}}} \rho A_{\mathrm{rm}} F_{\mathrm{rm}} ; \\
& Z_{12}=\frac{U_{\mathrm{tcp}}\left(F^{\prime} h_{\mathrm{pf}}+U_{\mathrm{tpf}}\right)}{2 \dot{m}_{\mathrm{f}} C_{\mathrm{f}}\left(U_{\mathrm{L} 2}+F^{\prime} h_{\mathrm{pf}}\right)} \\
& Z_{13}=P F_{1} P F_{2} \tau_{\mathrm{g}} \beta_{\mathrm{c}} \rho \frac{A_{\mathrm{am}}}{A_{\mathrm{rm}}} A_{\mathrm{rm}} F_{\mathrm{rm}} ; \\
& Z_{14}=\left(1+\left(1-\frac{A_{\mathrm{rc}} F_{\mathrm{rc}} U_{\mathrm{LC}}}{\dot{m}_{\mathrm{f}} C_{\mathrm{f}}}\right)\right) ; \quad Z_{15}=\left(1+K_{\mathrm{m}}\right)\left(\frac{1-K_{\mathrm{K}}^{N-1}}{1-K_{\mathrm{K}}}\right)
\end{aligned}
$$

$$
Z_{\mathrm{c}}=\left[Z_{9}+Z_{10}+Z_{11}+\left(Z_{12} Z_{13} Z_{14}\right)\right]
$$$$
(\alpha \tau)_{1, \text { eff }}=\left(\rho \alpha_{\mathrm{c}} \beta_{\mathrm{c}} \tau_{\mathrm{g}}-\eta_{\mathrm{m}} \rho\right) \frac{A_{\mathrm{am}}}{A_{\mathrm{rm}}} ; \quad(\alpha \tau)_{2, \mathrm{eff}}=\rho \alpha_{\mathrm{p}} \tau_{\mathrm{g}}^{2}\left(1-\beta_{\mathrm{c}}\right) \frac{A_{\mathrm{am}}}{A_{\mathrm{rm}}}
$$$$
(\alpha \tau)_{m, \mathrm{eff}}=(\alpha \tau)_{2, \mathrm{eff}}+P F_{1}(\alpha \tau)_{1, \mathrm{eff}}
$$

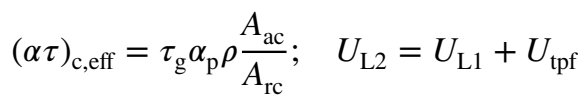$$
U_{\mathrm{Lm}}=\frac{h_{\mathrm{pf}} U_{\mathrm{L} 1}}{U_{\mathrm{L} 1}+F^{\prime} h_{\mathrm{pf}}+U_{\mathrm{tpf}}} ; \quad U_{\mathrm{Lc}}=\frac{h_{\mathrm{pf}} U_{\mathrm{tpa}}}{F^{\prime} h_{\mathrm{pf}}+U_{\mathrm{tpa}}} ;
$$$$
P F_{1}=\frac{U_{\mathrm{tcp}}}{U_{\mathrm{tca}}+U_{\mathrm{tcp}}} ; \quad P F_{2}=\frac{h_{\mathrm{pf}}}{U_{\mathrm{L} 1}+F^{\prime} h_{\mathrm{pf}}+U_{\mathrm{tpf}}}
$$$$
P F_{\mathrm{c}}=\frac{h_{\mathrm{pf}}}{F^{\prime} h_{\mathrm{pf}}+U_{\mathrm{tpa}}} ; \quad F_{\mathrm{rc}}=\frac{\dot{m}_{\mathrm{f}} C_{\mathrm{f}}}{A_{\mathrm{rc}} U_{\mathrm{Lc}}}\left(1-\exp \left(\frac{-\left(F^{\prime} U_{\mathrm{Lc}} A_{\mathrm{rc}}\right)}{\dot{m}_{\mathrm{f}} C_{\mathrm{f}}}\right)\right) ;
$$$$
F_{\mathrm{rm}}=\frac{\dot{m}_{\mathrm{f}} C_{\mathrm{f}}}{A_{\mathrm{rm}} U_{\mathrm{Lm}}}\left(1-\exp \left(\frac{-\left(F^{\prime} U_{\mathrm{Lm}} A_{\mathrm{rm}}\right)}{\dot{m}_{\mathrm{f}} C_{\mathrm{f}}}\right)\right)
$$$$
\left(A F_{\mathrm{R}}(\alpha \tau)\right)_{1}=\left[\left(P F_{\mathrm{c}}(\alpha \tau)_{\mathrm{c}, \mathrm{eff}} A_{\mathrm{rc}} F_{\mathrm{rc}}\right)\right.
$$

$$
\left.+\left(P F_{2}(\alpha \tau)_{\mathrm{m}, \mathrm{eff}} A_{\mathrm{rm}} F_{\mathrm{rm}}\left(1-\frac{A_{\mathrm{rc}} U_{\mathrm{Lc}} F_{\mathrm{rc}}}{\dot{m}_{\mathrm{f}} C_{\mathrm{f}}}\right)\right)\right]
$$$$
\left(A F_{\mathrm{R}} U_{\mathrm{L}}\right)_{1}=\left(A_{\mathrm{rc}} F_{\mathrm{rc}} U_{\mathrm{Lc}}\right)+A_{\mathrm{rm}} U_{\mathrm{Lm}} F_{\mathrm{rm}}\left(1-\frac{A_{\mathrm{rc}} U_{\mathrm{Lc}} F_{\mathrm{rc}}}{\dot{m}_{\mathrm{f}} C_{\mathrm{f}}}\right)
$$$$
U_{\text {tca }}=\left[\frac{1}{h_{\mathrm{o}}}+\frac{L_{\mathrm{g}}}{K_{\mathrm{g}}}\right]^{-1} ; \quad U_{\mathrm{tcp}}=\left[\frac{1}{h_{\mathrm{i}}}+\frac{L_{\mathrm{g}}}{K_{\mathrm{g}}}\right]^{-1} ;
$$$$
U_{\text {tpf }}=\left[\frac{L_{\mathrm{g}}}{K_{\mathrm{g}}}+\frac{1}{h_{\mathrm{pf}}}\right]^{-1} ; \quad U_{\mathrm{L} 1}=\frac{U_{\mathrm{tcp}} U_{\mathrm{tca}}}{U_{\mathrm{tcp}}+U_{\mathrm{tca}}}
$$$$
K_{\mathrm{m}}=1-\frac{A_{\mathrm{rm}} F_{\mathrm{rm}} U_{\mathrm{Lm}}}{\dot{m}_{\mathrm{f}} C_{\mathrm{f}}} ; \quad K_{\mathrm{K}}=1-\frac{\left(A F_{\mathrm{R}} U_{\mathrm{L}}\right)_{1}}{\dot{m}_{\mathrm{f}} C_{\mathrm{f}}} ;
$$$$
\left(A F_{\mathrm{R}} U_{\mathrm{L}}\right)_{\mathrm{m} 1}=A_{\mathrm{rm}} F_{\mathrm{rm}} U_{\mathrm{Lm}}
$$$$
T=\alpha_{\mathrm{w}} I(t)+\frac{h_{\mathrm{bw}} \alpha_{\mathrm{b}} I(t)}{h_{\mathrm{bw}}+h_{\mathrm{ba}}}+U_{\mathrm{bca}} T_{\mathrm{a}} ; \quad W=\frac{h_{2} A_{\mathrm{b}} h_{\mathrm{kg}} A_{\mathrm{g}}}{h_{\mathrm{kg}} A_{\mathrm{g}}+h_{2} A_{\mathrm{b}}} ;
$$$$
Y=\frac{h_{1} A_{\mathrm{g}}}{h_{1} A_{\mathrm{g}}+(U A)_{\mathrm{wgo}}}
$$ 


$$
\begin{aligned}
& Z=\frac{I(t)_{3}+h_{\mathrm{o}} T_{\mathrm{a}}}{(U A)_{\mathrm{w}, \mathrm{wf}}+h_{\mathrm{o}}}\left(1-\frac{1-\exp \left(-a_{2} l\right)}{a_{2} l}\right) ; \\
& U 1=T_{\mathrm{wfi}}\left(\frac{1-\exp \left(-a_{2} l\right)}{a_{2} l}\right) ; \quad V=\frac{I(t)_{\mathrm{A}}}{h_{1} A_{\mathrm{g}}+(U A)_{\mathrm{wgo}}} \\
& X=\frac{h_{2} A_{\mathrm{b}} \alpha_{\mathrm{b}} I(t) A_{\mathrm{g}}}{h_{2} A_{\mathrm{b}}+h_{\mathrm{kg}} A_{\mathrm{g}}} ; \quad I(t)_{\mathrm{A}}=\frac{h_{\mathrm{kg}} A_{\mathrm{g}} \alpha_{\mathrm{g}} I(t) A_{\mathrm{g}}}{h_{\mathrm{kg}} A_{\mathrm{g}}+h_{2} A_{\mathrm{b}}} ; \\
& U A_{\mathrm{wgo}}=\frac{h_{\mathrm{kg}} A_{\mathrm{g}} h_{2} A_{\mathrm{b}}}{h_{\mathrm{kg}} A_{g}+h_{2} A_{\mathrm{b}}} ; \quad U A_{\mathrm{w}, \mathrm{wf}}=\frac{h_{1}(U A)_{\mathrm{wgo}}}{h_{1} A_{\mathrm{b}}+(U A)_{\mathrm{wgo}}} \\
& h_{\mathrm{rw}}=\varepsilon \sigma\left[\frac{\left(T_{\mathrm{w}}+273\right)^{4}-\left(T_{\mathrm{a}}-273\right)^{4}}{\left(T_{\mathrm{w}}-T_{\mathrm{a}}\right)} ;\right. \\
& h_{\mathrm{ew}}=\left(16.27 \times 10^{-3} \times h_{\mathrm{cw}} \times\left(\frac{P_{\mathrm{w}}-P_{\mathrm{a}}}{T_{\mathrm{w}}-T_{\mathrm{a}}}\right)\right) \\
& h_{\mathrm{cw}}=0.884 \times\left[\left(T_{\mathrm{wfi}}-T_{\mathrm{a}}\right)+\frac{\left(P_{\mathrm{w}}-P_{\mathrm{a}}\right) \times\left(T_{\mathrm{a}}+273\right)}{\left(263.9 \times 10^{3}\right)-P_{\mathrm{w}}}\right] .
\end{aligned}
$$

\section{References}

1. Addams, L., Boccaletti, G., Kerlin, M., Stuchtey, M.: WRG (Water resource group): Charting our water future-economic framework to inform decision-making. Mc Kinsey \& Company, New York, Washington, DC (2009)

2. Della Porta, G.B.: Magiae Naturalis, Libri XX, Napoli (Italian translation, 1677, Napoli), English translation, New York, 1957, 1958, 1589 German translation, Nuremberg, 1713

3. El Sebaii, A.A.: Effect of wind speed on active and passive solar stills. Energy. Convers. Manag. 45, 1187-1204 (2004)

4. Tiwari, A.K., Tiwari, G.N.: Thermal modeling based on solar fraction and experimental study of the annual and seasonal performance of a single slope passive solar still: the effect of water depths. Desalination 207, 184-204 (2007)

5. Kabeel, A.E., Abdelgaied, M., Mahgoub, M.: The performance of a modified solar still using hot air injection and PCM. Desalination 379, 102-107 (2016)

6. Shanmugan, S., Rajamohan, P., Mutharasu, D.: Performance study on an acrylic mirror boosted solar distillation unit utilizing seawater. Desalination 230, 281-287 (2008)

7. Gupta, B., Shankara, P., Sharma, R., Baredar, P.: Performance enhancement using nano particles in modified passive solar still. Procedia Technol. 25, 1209-1216 (2016)

8. Abdelal, N., Taamneh, Y.: Enhancement of pyramid solar still productivity using absorber plates made of carbon fiber/CNTmodified epoxy composites. Desalination 419, 117-124 (2017)

9. Coventry, J.S.: Performance of a concentrating photovoltaic/thermal solar collector. Sol. Energy 78, 211-222 (2005)

10. Proell, M., Osgyan, P., Karrer, H., Brabec, C.J.: Experimental efficiency of a low concentrating CPC PVT flat plate collector. Sol. Energy 147, 463-469 (2017)

11. Rajaseenivasan, T., Raja, P.N., Srithar, K.: An experimental investigation on a solar still with an integrated flat plate collector. Desalination 347, 131-137 (2014)

12. Gaur, M.K., Tiwari, G.N.: Optimization of number of collectors for integrated PV/T hybrid active solar still. Appl. Energy 87, 1763-1772 (2010)
13. Arunkumar, T., Velraj, R., Denkenberger, D.C., Sathyamurthy, R., Kumar, K.V., Ahsan, A.: Productivity enhancements of compound parabolic concentrator tubular solar stills. Renew. Energy 88, 391-400 (2016)

14. Sharshir, S.W., Peng, G., Wu, L., Yang, N., Essa, F.A., Elsheikh, A.H., Mohamed, S.I.T., Kabeel, A.E.: Enhancing the solar still performance using nanofluids and glass cover cooling: experimental study. Appl. Therm. Eng. 113, 684-693 (2017)

15. Bassam, A.K.A.H.: Enhanced solar still performance using water film cooling of the glass cover. Desalination 107, 235-244 (1996)

16. Dhiman, N.K., Tiwari, G.N.: Effect of water flowing over the glass cover of a multi wick solar still. Energy Converg. Manag. 30, 245-250 (1990)

17. El-Samadony, Y.A.F., Kabeel, A.E.: Theoretical estimation of the optimum glass cover water film cooling parameters combinations of a stepped solar still. Energy 68, 744-750 (2014)

18. Sahota, L., Shyam, Tiwari, G.N.: Energy matrices, enviroeconomic and exergoeconomic analysis of passive double slope solar still with water based nanofluids. Desalination 409, 66-79 (2017)

19. Tiwari, G.N.: Enhancement of daily yield in a double basin solar still. Energy Conserv. Manag. 25(1), 49-50 (1985)

20. Elango, T., Murugavel, K.K.: The effect of water depth on the productivity for single and double basin slope glass solar still. Desalination 359, 82-91 (2015)

21. Pal, P., Yadav, P., Dev, R., Singh, D.: Performance analysis of modified basin type double slope multi-wick solar still. Desalination 422, 68-82 (2017)

22. Murugavel, K.K., Srithar, K.: Performance study on basin type double slope solar still with different wick materials and minimum mass of water. Renew. Energy 36, 612-620 (2011)

23. Sulttani, A.O.A., Ahsan, A., Rahman, A., Daud, N.N.N., Idrus, S.: Heat transfer coefficients and yield analysis of a double-slope solar still hybrid with rubber scrapers: an experimental and theoretical study. Desalination 407, 61-74 (2017)

24. Morad, M.M., El-Maghawry, H.A.M., Wasfy, K.I.: Improving the double slope solar still performance by using flat-plate solar collector and cooling glass cover. Desalination 373, 1-9 (2015)

25. Tellez, M.C., Figueroa, I.P., Juarez, A.S., Zayas, J.L.F.: Experimental study on the air velocity effect on the efficiency and fresh water production in a forced convective double slope solar still. Appl. Therm. Eng. 75, 1192-1200 (2015)

26. Singh, G., Kumar, S., Tiwari, G.N.: Design, fabrication and performance evaluation of hybrid photo-voltaic thermal (PVT) double slope active solar still. Desalination 277, 399-406 (2011)

27. Rahbar, N., Gharaiianc, A., Rashidia, S.: Exergy and economic analysis for a double slope solar still equipped by thermoelectric heating modules - an experimental investigation. Desalination 420, 106-113 (2017)

28. Singh, D.B., Tiwari, G.N.: Performance analysis of basin type solar stills integrated with $\mathrm{N}$-identical photovoltaic thermal (PVT) compound parabolic concentrator (CPC) collectors: a comparative study. Sol. Energy 142, 144-158 (2017)

29. Rabl, A.: Optical and thermal properties of compound parabolic concentrators (CPC). Sol. Energy 18, 497-511 (1976)

30. Fernandez, J., Chargoy, N.: Multistage, indirectly heated solar still. Sol. Energy 44(4), 215F (1990)

31. Kumar, S., Tiwari, G.N.: Estimation of internal heat transfer coefficients of a hybrid (PV/T active solar still. Sol. Energy 83, 1656-1667 (2009)

32. Jafarkazemi, F., Ahmadifard, E.: Energetic and exergetic evaluation of flat plate solar flat plate collector and hot air. Renew. Energy 56, 55-63 (2013)

33. Nag, P.K.: Basic and Applied Thermodynamics. Tata McGrawHill Education, New Delhi, India (2002)

34. Tiwari, G.N., Mishra, R.K.: Advanced Renewable energy sources. Royal Chemistry of Society, Cambridge (2012) 
35. Kumar, S., Tiwari, G.N.: Life cycle cost analysis of single slope hybrid (PV/T) active solar still. Appl. Energy 86, 1995-2004 (2009)

36. Onovwiona, H.I., Ugursal, V.I.: Residential cogeneration systems: review of the current technology. Renew. Sustain. Energy Rev. 10, 389-431 (2006)
37. Tiwari, G.N.: Solar Energy—Fundamentals. Modelling and Applications. Narosa Publishing House, New-Delhi, India, Design (2002)

Publisher's Note Springer Nature remains neutral with regard to jurisdictional claims in published maps and institutional affiliations. 